\title{
Entire minimizers of Allen-Cahn systems with sub-quadratic potentials
}

\author{
Nicholas D. Alikakos*, Dimitrios Gazoulis ${ }^{\dagger}$, Arghir Zarnescu ${ }^{\ddagger \S} \mathbb{1}$
}

Dedicated to Pavol Brunovsky, a man of brilliance and very high morality

\begin{abstract}
We study entire minimizers of the Allen-Cahn systems. The specific feature of our systems are potentials having a finite number of global minima, with sub-quadratic behaviour locally near their minima. The corresponding formal Euler-Lagrange equations are supplemented with free boundaries.

We do not study regularity issues but focus on qualitative aspects. We show the existence of entire solutions in an equivariant setting connecting the minima of $W$ at infinity, thus modeling many coexisting phases, possessing free boundaries and minimizing energy in the symmetry class. We also present a very modest result of existence of free boundaries under no symmetry hypotheses. The existence of a free boundary can be related to the existence of a specific sub-quadratic feature, a dead core, whose size is also quantified.
\end{abstract}

\section{Introduction and Main Results}

In this note we consider minimizers in the whole space $\mathbb{R}^{n}$ for the functional

$$
J(u)=\int \frac{1}{2}|\nabla u|^{2}+W(u) d x
$$

*Department of Mathematics, University of Athens (EKPA), Panepistemiopolis, 15784 Athens, Greece (nalikako@math.uoa.gr )

${ }^{\dagger}$ Department of Mathematics and Applied Mathematics, University of Crete, 70013 Heraklion, Greece (dgazoulis@math.uoa.gr )

${ }^{\ddagger}$ IKERBASQUE, Basque Foundation for Science, Plaza Euskadi 548009 Bilbao, Bizkaia, Spain (azarnescu@bcamath.org)

§BCAM, Basque Center for Applied Mathematics, Mazarredo 14, E48009 Bilbao, Bizkaia, Spain

I "Simion Stoilow" Institute of the Romanian Academy, 21 Calea Grivitei, 010702 Bucharest, Romania. 
with $u: \mathbb{R}^{n} \rightarrow \mathbb{R}^{m}$.

We take $W \geq 0$ and $\{W=0\}=\left\{a_{1}, \ldots, a_{N}\right\}:=A$, for some distinct points $a_{1}, \ldots, a_{N} \in$ $\mathbb{R}^{m}$ that can physically model the phases of a substance that can exist in $N \geq 2$ equally preferred states.

We assume that

$$
\liminf _{|z| \rightarrow \infty} W(z)>0
$$

If $W$ is smooth then the first derivatives vanish at the minimum points and the generic local behaviour near such a minimum, say $a_{i}$, is locally of quadratic nature, of the type $\left|u-a_{i}\right|^{2}$. The minimizers satisfy the Euler-Lagrange system

$$
\Delta u-W_{u}(u)=0
$$

We are interested in the class of solutions that connect in some way the phases or a subset of them. The scalar case $m=1$ has been extensively studied with $N=2$ that is the natural choice. The reader may consult [20], [37], [41] where further references can be found. A well known conjecture of De Giorgi (1978) and its solution about thirty years later, played a significant role in the development of a large part of this work.

The vector case $m \geq 2$ by comparison has been studied very little. We note that for coexistence of three or more phases a vector order parameter is necessary and so there is physical interest for the system.

For $m \geq 2$, (1.3) has been mainly studied in the class of equivariant solutions with respect to reflection groups beginning with [13] and later [27] and significantly extended and generalized in various ways [6], [3], [24], [4], [7], [11]. We refer to [1] where existence under symmetry is covered and where more references can be found.

Degenerate, super-quadratic behavior at the minima has also been considered for (1.3), $m=1$, in [12, [21].

The focus of our work will be on going beyond this classical setting and explore the phenomena that are associated having sub-quadratic behaviour at the minima. Specifically, our potentials are modelled near their minima $a \in A$ after $|u-a|^{\alpha}$, for $0<\alpha<2$. Furthermore we will consider also the limiting case $\alpha=0$ (that appears in a $\Gamma$-limit setting as $\alpha \rightarrow 0$ ). Formally, the minimizers solve certain free boundary problems:

1. For $\alpha \in(0,2)$ :

$$
\left\{\begin{array}{l}
\Delta u=W_{u}(u) \text { for }\{u(x) \notin A\} \\
|\nabla u|^{2}=0 \text { for } \partial\{u(x) \notin A\}
\end{array}\right.
$$


2. For $\alpha=0$ :

$$
\left\{\begin{array}{l}
\Delta u=0 \text { for }\{u(x) \notin A\} \\
|\nabla u|^{2}=2 \text { for } \partial\{u(x) \notin A\}
\end{array}\right.
$$

In Appendix B we give a formal justification of these, that can be made rigorous with suitable regularity results, [8]. We note that for $\alpha=2$, Corollary 3.1 p.92 in [1] states that if both $W(u(x))=0$ and $|\nabla u(x)|^{2}=O\left(W(u(x))\right.$ then $u \equiv a_{i}$. This latter condition holds in the scalar case, $m=1$, by the Modica inequality. Hence for $\alpha=2, m=1$ we have $\partial\{u(x) \notin A\}=\emptyset$. Thus a free boundary may be expected only in the non smooth case. The reason is rather simple and can be traced back to the non-uniqueness of the trivial solution of the ODE $u^{\prime}=\frac{2}{2-\alpha} C^{\frac{\alpha}{2}} u^{\frac{\alpha}{2}}$ that describes the behavior of the one-dimensional solutions (connections) near the minimum of $W$ of (1.4), (1.5).

Thus we focus on the range $0 \leq \alpha<2$. An important special case of the potentials we consider is given, for the set of minima $A=\left\{a_{1}, \ldots, a_{N}\right\}$ by

$$
W^{\bar{\alpha}}(u)=\prod_{k=1}^{N}\left|u-a_{k}\right|^{\alpha_{k}} \quad, \bar{\alpha}=\left(\alpha_{1}, \ldots, \alpha_{N}\right) ; 0<\alpha_{k}<2, \forall k \in\{1, \ldots, N\}
$$

More generally, motivated by the form of $W$ in (1.6), we assume:

(H1)

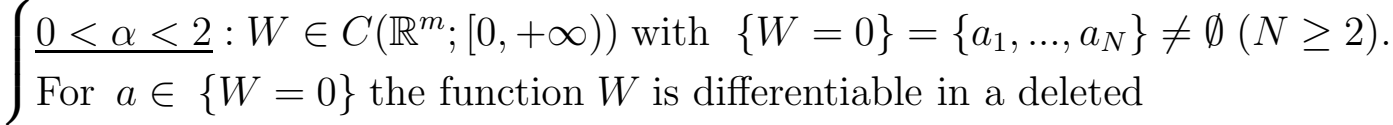

$$
\begin{aligned}
& \left\{\begin{array}{l}
\text { neighborhood of } a \text { and satisfies } \frac{d}{d \rho} W(a+\rho \xi) \geq \alpha C^{*} \rho^{\alpha-1}, \forall \rho \in\left(0, \rho_{0}\right], \\
\forall \xi \in \mathbb{R}^{m}:|\xi|=1, \text { for some constants } \rho_{0}>0, C^{*}>0 \text { independent of } \alpha .
\end{array}\right. \\
& \left\{\begin{array}{l}
\underline{\alpha=0}:\{W=0\}=\left\{a_{1}, \ldots, a_{N}\right\}:=A, W(u):=W^{0}(u):=\chi_{\left\{u \in S_{A}\right\}} \\
S_{A}:=\left\{\sum_{i=1}^{N} \lambda_{i} a_{i}, \lambda_{i} \in[0,1), \forall i=1, \ldots, N, \sum_{i=1}^{N} \lambda_{i}=1, N=m+1\right\}
\end{array}\right. \\
& \text { We assume that the simplex } S_{A} \text { is nondegenerate, that is the vectors } \\
& \left\{a_{2}-a_{1}, \ldots, a_{m+1}-a_{1}\right\} \text { are linearly independent and } m \geq 2 \text {. }
\end{aligned}
$$

Clearly $W^{\bar{\alpha}}$ in (1.6) satisfy (H1) $(0<\alpha<2)$.

We are primarily interested in bounded minimizers defined on $\mathbb{R}^{n}$. We note in passing that the only critical points of $J_{\mathbb{R}^{n}}, n \geq 2$, with bounded energy are trivial [2]. A minimizer $u$, by definition minimizes energy subject to its Dirichlet values on any open, bounded $\Omega \subset \mathbb{R}^{n}$. More precisely, 
Definition 1.1. Let $\mathcal{O} \subset \mathbb{R}^{n}$ open. A map $u \in W_{\text {loc }}^{1,2}\left(\mathcal{O}, \mathbb{R}^{m}\right) \cap L^{\infty}\left(\mathcal{O} ; \mathbb{R}^{m}\right)$ is called a minimizer of the energy functional $J$ defined in (1.1) if

$$
J_{\Omega}(u+v) \geq J_{\Omega}(u), \text { for } v \in W_{0}^{1,2}\left(\Omega, \mathbb{R}^{m}\right) \cap L^{\infty}\left(\Omega ; \mathbb{R}^{m}\right)
$$

for every open bounded Lipschitz set $\Omega \subset \mathcal{O}$, with $J_{\Omega}$ denoting the value of the integral in (1.1) when integrating over the domain $\Omega$.

The case, $\alpha=0$ for $m=1$ was introduced and extensively studied by Caffarelli and his collaborators, with particular attention to the optimal regularity of the solution and to the regularity of the free boundary. These are important classical results that can be found for example in the books [16] or [33]. There is recent interest in the vector case for free boundary problems. We mention below two papers which relate to our work and where additional references can be found.

In [17] the authors study minimizers of the functional

$$
\int_{\Omega}\left(\frac{1}{2}|\nabla u|^{2}+Q^{2}(x) \chi_{\{|u|>0\}}\right) d x
$$

with $u: \Omega \subset \mathbb{R}^{n} \rightarrow \mathbb{R}^{m}, u_{i} \geq 0, \Omega$ bounded and $u=g$ on $\partial \Omega$. This corresponds to a cooperative system, and is a one-phase Bernoulli-type problem. On the other hand, our nonlinearity is of the competitive kind and our problem is a two-phase Bernoulli-type problem.

In [29] the functional that is studied is

$$
\sum_{i=1}^{m} \int_{\Omega} \frac{1}{2}\left|\nabla u_{i}\right|^{2}+\Lambda \mathcal{L}^{n}\left(\cup_{i=1}^{m}\left\{u_{i} \neq 0\right\}\right) d x
$$

with $u_{i}=\phi_{i}$ on $\partial \Omega$. This is a two-phase type problem and it is quite close to our functional for $\alpha=0$.

The emphasis in these works is on the regularity of the solution and of the free boundary, while the existence of the free boundary is forced by the Dirichlet condition on $\partial \Omega$, and is not an issue in that context.

For stating our main results we need some algebraic preliminaries.

A reflection point group $G$ is a finite subgroup of the orthogonal group whose elements $g$ fix the origin. We will be assuming for simplicity that $m=n$ (the general case is presented in [1], Chapter 7), and that $G$ acts both on the domain space $\mathbb{R}^{n}$ and the target space $\mathbb{R}^{m}$. A map $u: \mathbb{R}^{n} \rightarrow \mathbb{R}^{n}$ is said to be equivariant with respect to the action of $G$, simply equivariant, if

$$
u(g x)=g u(x) \quad, \forall g \in G, x \in \mathbb{R}^{n}
$$


A reflection $\gamma \in G$ is a map $\gamma: \mathbb{R}^{n} \rightarrow \mathbb{R}^{n}$ of the form

$$
\gamma x=x-2\left(x \cdot n_{\gamma}\right) n_{\gamma}, \text { for } x \in \mathbb{R}^{n}
$$

for some unit vector $n_{\gamma} \in \mathbb{S}^{n-1}$ which aside from its orientation is uniquely determined by $\gamma$. The hyperplane

$$
\pi_{\gamma}=\left\{x \in \mathbb{R}^{n}: x \cdot n_{\gamma}=0\right\}
$$

is the set of the points that are fixed by $\gamma$. The open half space $\mathcal{S}_{\gamma}^{+}=\left\{x \in \mathbb{R}^{n}: x \cdot n_{\gamma}>0\right\}$ depends on the orientation of $n_{\gamma}$. We let $\Gamma \subset G$ denote the set of all reflections in $G$. Every finite subgroup of the orthogonal group $O\left(\mathbb{R}^{n}\right)$ has a fundamental region, that is a subset $F \subset \mathbb{R}^{n}$ with the following properties:

1. $F$ is open and convex,

2. $F \cap g F=\emptyset$ for $I \neq g \in G$, where $I$ is the identity,

3. $\mathbb{R}^{n}=\cup\{g \bar{F}: g \in G\}$.

The set $\cup_{\gamma \in \Gamma} \pi_{\gamma}$ divides $\mathbb{R}^{n} \backslash \cup_{\gamma \in \Gamma} \pi_{\gamma}$ in exactly $|G|$ congruent conical regions. Each one of these regions can be identified with the fundamental region $F$ for the action of $G$ on $\mathbb{R}^{n}$. We assume that the orientations of $n_{\gamma}$ are such that $F \subset \mathcal{S}_{\gamma}^{+}$and we have

$$
F=\cap_{\gamma \in \Gamma} \mathcal{S}_{\gamma}^{+}
$$

Given $a \in \mathbb{R}^{n}$, the stabilizer of $a$, denoted by $G_{a} \subset G$ is the subgroup of the elements $g \in G$ that fix $a$ :

$$
G_{a}=\{g \in G: g a=a\} .
$$

We now introduce two more hypotheses:

(H2)(symmetry) The potential $W$ is invariant under a reflection (point) group $G$ acting on $\mathbb{R}^{n}$, that is

$$
W(g u)=W(u) \text { for all } g \in G \text { and } u \in \mathbb{R}^{n} .
$$

Moreover we assume (1.2).

(H3)(Location and number of global minima) Let $F \subset \mathbb{R}^{n}$ be a fundamental region of $G$. We assume that $\bar{F}$ contains a single global minimum of $W$ say $a_{1} \neq 0$, and let $G_{a_{1}}$ be the stabilizer of $a_{1}$. Setting $D:=\operatorname{Int}\left(\cup_{g \in G_{a_{1}}} g \bar{F}\right), a_{1}$ is also the unique global minimum of $W$ in the region $D$. 
Notice that, by the invariance of $W$, Hypothesis (H3) implies that the number of minima of $W$ is

$$
N=\frac{|G|}{\left|G_{a_{1}}\right|},
$$

where $|\cdot|$ stands for the number of elements.

We can now state our first main result.

Theorem 1. $(0<\alpha<2)$ Under hypothesis (H1)-(H3), there exists an equivariant minimizer $u$ of $J, u: \mathbb{R}^{n} \rightarrow \mathbb{R}^{n}$, such that

1. $\left|u(x)-a_{1}\right|=0$ for $x \in D$ and $d(x, \partial D) \geq d_{0}$, where $d_{0}$ a positive constant depending on $\|u\|_{L^{\infty}\left(\mathbb{R}^{n}, \mathbb{R}^{n}\right)}, \quad C^{*}$ and $\alpha\left(d_{0} \rightarrow+\infty\right.$ as $\left.\alpha \rightarrow 2\right)$.

2. $u(\bar{F}) \subset \bar{F}, u(\bar{D}) \subset \bar{D}$ (positivity).

Hence by equivariance the statements above hold for all $a_{i}, i=1, \ldots, N$, in the respective copy of $D$.

Remark 1.2. In [8] it is shown that $u \in C_{\text {loc }}^{2, \alpha-1}$ for $\alpha \in(1,2), u \in C_{\text {loc }}^{1, \gamma}$ for any $\gamma \in(0,1)$ and $u \in C^{1, \frac{\alpha}{1-\alpha}}$ for $\alpha \in(0,1)$. The regularity for $\alpha \in(0,1)$ is optimal. In Lemma 2.1 we establish the (suboptimal) estimate $|u|_{C^{\beta}}<\infty$ (any $\left.\beta \in(0,1)\right)$ that holds for all $\alpha \in[0,2)$ which is sufficient for our purposes. We revisit this point also later.

The analog of Theorem 1 for $\alpha=2, W \in C^{2}$ was established in a series of papers by the first author and G.Fusco. It can be found in [1] (Theorem 6.1) where detailed references are given. The main difference with Theorem 1 above is that the condition $\left|u(x)-a_{1}\right|=0$ for $x \in D, d(x, D) \geq d_{0}$, is replaced by $\left|u(x)-a_{1}\right| \leq K e^{-k d(x, \partial D)}, x \in D$, where $k, K$ are positive constants. In that context the minimizer $u$ is a classical solution of (1.3) while in the present context $u$ is a weak $W_{l o c}^{1,2}$ solution of (1.3) in the complement of the free boundary $\partial\{u(x) \notin A\}$. The theorem in the smooth case is utilized in our proof of Theorem 1 where we are constructing a minimizer with the positivity property via a $C^{2}$ regularization of the potential. We thus bypass the gradient flow argument used in the proof of the $\alpha=2$ case in [1] that would be problematic in the present setting. The role of positivity can be seen in the following proposition, which does not presuppose symmetry.

Proposition 1. $(0<\alpha<2)$ (i) Assume that $W$ as in (H1) above, and $u$ a bounded minimizer of $J, u: \mathbb{R}^{n} \rightarrow \mathbb{R}^{m},\|u\|_{L^{\infty}\left(\mathbb{R}^{n}, \mathbb{R}^{m}\right)}<\infty$. Moreover, let $\mathcal{O} \subset \mathbb{R}^{n}$ open, assume that

$$
d(u(\mathcal{O}),\{W=0\} \backslash\{a\}) \geq k>0
$$


$d$ the Euclidean distance, $\mathrm{k}$ constant.

Then given $q \in\left(0,\|u\|_{L^{\infty}\left(\mathbb{R}^{n}, \mathbb{R}^{m}\right)}\right), \exists r_{q}>0$ such that

$$
B_{r_{q}}\left(x_{0}\right) \subset \mathcal{O} \Rightarrow\left|u\left(x_{0}\right)-a\right|<q
$$

(ii) Let further $0<2 q \leq \rho_{0}(\operatorname{cfr}(\mathbf{H 1}))$. Then there exists an explicit constant $\hat{C}=\hat{C}(\alpha, n)>$ 0 (see (3.18) $, \lim _{\alpha \rightarrow 2} \hat{C}(\alpha, n)=\infty, \lim _{\alpha \rightarrow 0} \hat{C}(\alpha, n)=\infty$ ), such that

$$
B_{\hat{C} q^{-\alpha}\left(x_{0}\right)} \subset \mathcal{O} \Rightarrow u(x) \equiv a \text {, in } B_{\frac{\hat{C}}{2} q^{-\alpha}\left(x_{0}\right)}
$$

Remark 1.3. Part (i) of Proposition 1 holds for $\alpha=2$, and is a result obtained in [23]. It can be found also in [1] Theorem 5.3. Note that positivity allows the application of this with $\mathcal{O}=D$, since the solution in $D$ stays away from all the minima except one. This reveals the nature of (H3).

Part (ii) is utilizing a "Dead Core" estimate (Lemma 2.5 below) which shows that for a function $v \in W^{1,2}\left(B_{R}\left(x_{0}\right)\right)$

$$
\left\{\begin{array}{l}
\Delta v \geq c^{2} v^{\frac{\alpha}{2}}, \text { weakly in } W^{1,2}\left(B_{R}\left(x_{0}\right)\right) \\
0 \leq v \leq \delta, \delta>0 \text { sufficiently small depending on } c
\end{array}\right.
$$

Then if

$$
\left\{\begin{array}{l}
\operatorname{dist}\left(y_{0}, \partial B_{R}\left(x_{0}\right)\right)>R_{0} \Rightarrow \\
v\left(y_{0}\right)=0 \text { for } R>R_{0}=\frac{\sqrt{n(n+2)}}{\left(1-\frac{\alpha}{2}\right) c} \delta^{\frac{2-\alpha}{4}}, \alpha \in(0,2)
\end{array}\right.
$$

"Dead Core" regions are sets where the solution is constant.

The first appearance of such a situation was in [16], 34, followed by more in depth study in [39].

Proposition 2. $(\alpha=0)$ Let

$$
J(u)=\int\left(\frac{1}{2}|\nabla u|^{2}+\chi_{A^{c}}(u)\right) d x
$$

where $A:=\{W=0\}=\left\{a_{1}, \ldots, a_{N}\right\} \subset \mathbb{R}^{m}(N \geq 2), A^{c}=\mathbb{R}^{m} \backslash A$. Let $u$ be a nonconstant minimizer, $u: \mathbb{R}^{n} \rightarrow \mathbb{R}^{m},\|u\|_{L^{\infty}\left(\mathbb{R}^{n}, \mathbb{R}^{m}\right)}<\infty$. Suppose that for some $a_{i} \in A$ we have

$$
d\left(u\left(B_{R}\left(x_{0}\right)\right),\{W=0\} \backslash a_{i}\right)>0
$$

Then

$$
\mathcal{L}^{n}\left(\left\{u=a_{i}\right\} \cap B_{R}\left(x_{0}\right)\right) \geq c R^{n}, R \geq R_{0}
$$

for some constant $c>0$ independent of $R$. 
What about existence of minimizer defined on $\mathbb{R}^{n}$ possessing a free boundary and without any symmetry assumptions? This is a difficult open problem for the coexistence of three or more phases. We have the following simple result in this direction.

Proposition 3. $(\alpha=0)$ Consider the functional

$$
J(u)=\int\left(\frac{1}{2}|\nabla u|^{2}+\chi_{A^{c}}(u)\right) d x
$$

where $A=\left\{a_{1}, \ldots, a_{N}\right\}$ distinct points in $\mathbb{R}^{m}, A^{c}=\mathbb{R}^{m} \backslash A$.

Let $u: \mathbb{R}^{n} \rightarrow \mathbb{R}^{m}$ be a nonconstant minimizer with $\|u\|_{L^{\infty}\left(\mathbb{R}^{n}, \mathbb{R}^{m}\right)}<\infty$ and $x_{0} \in \mathbb{R}^{n}$, arbitrary and fixed. Then there exist an $R_{0}>0$ and at least two distinct points $a_{i} \neq a_{j}$ in $A$, such that the following estimates hold:

$$
\begin{gathered}
\mathcal{L}^{n}\left(\overline{B_{R}\left(x_{0}\right)} \cap\left\{u(x)=a_{k}\right\}\right) \geq c_{k} R^{n}, R \geq R_{0}, k=i, j \\
\left\|\partial\left\{u(x)=a_{k}\right\}\right\|\left(B_{R}\left(x_{0}\right)\right) \geq \hat{c}_{k} R^{n-1}, R \geq R_{0}, k=i, j
\end{gathered}
$$

where $c_{k}, \hat{c}_{k}$ are positive constants, independent of $x_{0}$ and $R$ (but depending on $u$ ). $\|\partial E\|$ stands for the perimeter measure of the set $E$ and $\|\partial E\|\left(B_{R}\left(x_{0}\right)\right)$ denotes the perimeter of $E$ in $B_{R}\left(x_{0}\right)$ (see for instance [22]).

Remark 1.4. Proposition 3 holds for the whole range of potentials $0<\alpha<2$ defined in (H1) but with a significantly harder proof 8 .

The natural way of constructing entire solutions $u$ to (1.3) without symmetry requirements is by minimizing over balls $B_{R}$ with appropriate boundary conditions forcing the phases on $B_{R}$ :

$$
\min J_{B_{R}}(v), v=g_{R}, \text { on } \partial B_{R},
$$

and taking the limit along subsequences of minimizers $u_{R}$

$$
u=\lim _{R \rightarrow \infty} u_{R}
$$

Remark 1.5. The result from Proposition 3 holds for the symmetric case as in Theorem 1 for $\alpha=0$, and provides some quantitative information on the Dead Core. We have not been able to establish the exact analog of Theorem 1 for $\alpha=0$.

Proposition 4. $(\alpha=0)$ Under the hypothesis (H1)-(H3) and $N=m+1$, there exist a nontrivial equivariant minimizer of $J(u)=\int\left(\frac{1}{2}|\nabla u|^{2}+\chi_{\left\{u \in S_{A}\right\}}\right) d x, u: \mathbb{R}^{n} \rightarrow \mathbb{R}^{n}$, such that 1. $u(\bar{F}) \subset \bar{F}, u(\bar{D}) \subset \bar{D}$ (positivity).

2. $\mathcal{L}^{n}\left(D_{R} \cap\left\{u=a_{1}\right\}\right) \geq c R^{n}, R \geq R_{0}$, where $D_{R}=D \cap B_{R}(0)$ ( $D$ from (H3)).

3. $\mathcal{L}^{n}\left(D_{R} \cap\left\{u \neq a_{1}\right\}\right) \leq C R^{n-1}, R \geq R_{0}$. 
A convenient hypothesis guaranteeing $\|u\|_{L^{\infty}}<\infty$ is 1

$$
\left\{\begin{array}{l}
W_{u}(u) \cdot u \geq 0, \text { for }|u| \geq M, \text { some } M \\
\left|g_{R}\right| \leq M
\end{array}\right.
$$

The existence of one-dimensional minimizers $\left(u: \mathbb{R} \rightarrow \mathbb{R}^{n}\right.$, i.e. connections) for $\alpha \in(0,2)$, can be obtained by Theorem 2.1, p.34 in [1]. For the $\alpha=0$ case, where $W$ is a characteristic function, one-dimensional minimizers are affine maps connecting the phases. More precisely,

$$
u(x)=\left\{\begin{array}{l}
a_{1}, x<-L \\
a_{2}, x>L \\
\frac{a_{2}-a_{1}}{2 L} x+\frac{a_{1}+a_{2}}{2}, x \in[-L, L]
\end{array}\right.
$$

and by minimality one can see that $L=\frac{\left|a_{2}-a_{1}\right|}{2 \sqrt{2}}$, which is formally what we expect from the free boundary condition $|\nabla u|^{2}=2$ (see (1.5)) .

The basic question of course is whether a nontrivial minimizer $u$ connecting the phases can be constructed. We know from the work on the De Giorgi referred above conjecture that for $m=1$, and in low dimensions, any such minimizer will depend on a single variable, and so in a sense is trivial. For the system we expect otherwise, and indeed this was shown to be the case in the equivariant setting and for smooth potentials, in the book [1].

There are a few tools that we utilize in the sequel that because of their independent interest we mention explicitly.

\section{The Basic Estimate}

For minimizers, $0 \leq \alpha<2$ satisfying $|u(x)| \leq M, x \in \mathbb{R}^{n}$ we have that there exists $r_{0}>0$ such that for any $x_{0} \in \mathbb{R}^{n}$

$$
J_{B_{r}\left(x_{0}\right)}(u) \leq C_{0} r^{n-1}, r \geq r_{0}>0,
$$

$C_{0}>0$ constant, independent of $u$, but depending on $M$.

For $\alpha \in[1,2)$ elliptic theory applied to (1.3) implies $\|\nabla u\|_{L^{\infty}}<\infty$, and (1.23) follows easily (cfr. [1] Lemma 5.1). For $\alpha \in[0,1)$, and $m=1$, it is already mentioned in [14]. We prove it in Lemma 2.2. The estimate (1.23) is utilized in the proof of Proposition 3, and also in the proof of Proposition 1 on which Part 1 of Theorem 1 is based. Finally (1.23) is also

\footnotetext{
${ }^{1}$ For $\Omega \subset \mathbb{R}^{n}$ open, by linear elliptic theory $u \in C^{2}\left(\Omega ; \mathbb{R}^{m}\right)$. Set $v=|u|^{2}$, then $\Delta v=2 W_{u}(u) \cdot u+2|\nabla u|^{2}>$ 0 , for $u>M$. Hence $\max |u|^{2} \leq M$ if $v$ attains its max in the interior of $\Omega$.
} 
utilized in the proof of the Density Estimate that we discuss below.

The Density Estimate

For minimizers $u$ of the functional $J$ in (1.1), $0 \leq \alpha<2$ satisfying $|u(x)| \leq M$, we have

$$
\left\{\begin{array}{l}
\mathcal{L}^{n}\left(B_{r_{0}}\left(x_{0}\right) \cap\{|u-a|>\lambda\}\right) \geq \mu_{0}>0 \Rightarrow \\
\mathcal{L}^{n}\left(B_{r}\left(x_{0}\right) \cap\{|u-a|>\lambda\}\right) \geq C r^{n} \quad, r \geq r_{0}
\end{array}\right.
$$

$C=C\left(\mu_{0}, \lambda\right)$

This is an important estimate of Caffarelli and Cordoba [14 established in the scalar case $m=1$, and extended to the vector case by the first author and G.Fusco. We refer to [1] Theorem 5.2, where detailed references can be found. The proof in [1] has a gap for $0 \leq \alpha<1$ since it is utilizing (1.23) that was taken for granted then but proved in the present paper.

The Hölder Estimate

For minimizers $u$ of the functional $J$ in (1.1), $0 \leq \alpha<2$, satisfying $|u(x)|<M, x \in \mathbb{R}^{n}$, we have the estimate

$$
|u(x)-u(y)| \leq C|x-y| \ln \left(|x-y|^{-1}\right) \quad, \forall x, y,|x-y| \leq \frac{1}{2}
$$

which implies $u \in C^{\beta}\left(\mathbb{R}^{n}, \mathbb{R}^{m}\right), \forall \beta \in(0,1), C=C(M)$, that has already be mentioned.

This is established in [10] for $m=1$ and $\alpha=0$. We give a detailed proof in Lemma 2.1. It is utilized in several places. For example in establishing Proposition 1 (i) we proceed by a contradiction argument that invokes the Density Estimate. Here uniform continuity is essential, and is provided by (1.25). It is also instrumental for the derivation of the Basic Estimate (1.23).

The Hölder continuity is also needed in the proof of the Containment result presented in Appendix A, that we now describe.

\section{The Containment}

This states that for the special potentials

$$
W(u)=\left\{\begin{array}{l}
W^{\bar{\alpha}}(u):=\prod_{k=1}^{m+1}\left|u-a_{k}\right|^{\alpha_{k}}, \bar{\alpha}=\left(a_{1}, \ldots, a_{m+1}\right), 0<a_{k}<2 \\
W^{0}(u):=\chi_{A^{c}}(u), A=\left\{a_{1}, \ldots, a_{m+1}\right\}
\end{array}\right.
$$

where the vectors $\left\{a_{2}-a_{1}, \ldots, a_{m+1}-a_{1}\right\}$ are linearly independent in $\mathbb{R}^{m}$, critical points of $J(u)=\int\left(\frac{1}{2}|\nabla u|^{2}+W(u)\right) d x, u: \mathbb{R}^{n} \rightarrow \mathbb{R}^{n},|u(x)|<M$, map $\mathbb{R}^{n}$ inside the closure 
of the convex hull of $A, \overline{c o}(A)$. This result was obtained jointly by the first author and P.Smyrnelis, in unpublished work. Its proof requires uniform continuity, and so for $\alpha \in(0,1)$ we need to restrict ourselves to minimizers for which (1.25) holds.

This result shows that $J^{0}$ is in some natural way the limit of $J^{\bar{\alpha}}$, as $\bar{\alpha} \rightarrow 0$, and actually we establish a $\Gamma$-limit type relationship in Lemma 2.9.

This paper is structured as follows.

In section 2 we state and prove various Lemmas already mentioned in the introduction.

In section 3 we give the proofs of Theorem 1, Propositions 1, 2 and 3.

In Appendix $\mathrm{A}$ we state and prove the containment result, and in Appendix $\mathrm{B}$ we give a formal argument, taken essentially from [1], that explains the free boundary conditions in (1.4) and (1.5).

Acknowledgements We are greateful to Panayotis Smyrnelis for his interest in this work and his numerous comments that improved the paper. AZ would like to thank Prof. Luc Nguyen for pointing out the log-estimate argument in the proof of Lemma 2.1. Finally we would like to thank Zhiyuan Geng for introducing us to free boundary problems.

The work of A.Z. is supported by the Basque Government through the BERC 2018-2021 program, by Spanish Ministry of Economy and Competitiveness MINECO through BCAM Severo Ochoa excellence accreditation SEV-2017-0718 and through project MTM2017-82184R funded by (AEI/FEDER, UE) and acronym "DESFLU".

D.G. would like to acknowledge support of this work by the project "Innovative Actions in Environmental Research and Development (PErAn" (MIS 5002358) which is implemented under the "Action for the Strategic Development on the Research and Technological Sector", funded by the Operational Programme "Competitiveness, Entrepreneurship and Innovation" (NSRF 2014-2020) and co-financed by Greece and the European Union (European Regional Development Fund).

N.D.A. held a BCAM visiting fellowship in the fall of 2019 during which some of the results of the present paper were established; also would like to thank his host, Arghir Zarnescu and the people in the institute for their hospitality. 


\section{Basic Lemmas}

\section{$2.1 \quad$ Regularity of $u$}

We will prove a logarithmic estimate for bounded minimizers, following closely the proof of Theorem 2.1 in [10] (see also Lemma 2 in [17]). We have:

Lemma 2.1. ( $0 \leq \alpha<2$, Hölder Continuity)

Let $u: \mathbb{R}^{n} \rightarrow \mathbb{R}^{m}$ a minimizer of $J,|u(x)|<M, W$ satisfying (H1) for $0<\alpha<2$ and $W=\chi_{A^{c}}(u)$ for $\alpha=0$. Then there exists constant $C=C(M)$, such that

$$
|u(x)-u(y)| \leq C|x-y| \ln \left(|x-y|^{-1}\right) \quad, \forall x, y,|x-y| \leq \frac{1}{2}
$$

In paricular, $u \in C^{\beta}\left(\mathbb{R}^{n} ; \mathbb{R}^{m}\right), \forall \beta \in(0,1)$.

Proof. We restrict ourselves to $0 \leq \alpha<1$, since the result follows immediately for $\alpha \in[1,2]$ by linear elliptic theory. We begin with the case $0<\alpha<1$.

For an arbitrary $B_{r}\left(x_{0}\right)$ let $v_{r}$ be the harmonic function equal to $u$ on $\partial B_{r}$. Then by the maximum principle $v_{r}$ is also bounded and taking into account the specific form of the potential (1.6) we have that there exists an $M$ such that:

$$
|u(x)|,\left|v_{r}(x)\right|,\left|W^{\bar{\alpha}}(u(x))\right|,\left|W^{\bar{\alpha}}\left(v_{r}(x)\right)\right| \leq M, \forall x \in B_{r}\left(x_{0}\right), \alpha \in[0,1]
$$

Then using the minimality of $u$ and the non-negativity of the potentials $W^{\bar{\alpha}}$ together with (2.2) we have:

$$
\begin{aligned}
\int_{B_{r}}|\nabla u(x)|^{2} d x & \leq \int_{B_{r}}|\nabla u(x)|^{2}+W^{\bar{\alpha}}(u(x)) d x \leq \int_{B_{r}}\left|\nabla v_{r}(x)\right|^{2}+W^{\bar{\alpha}}\left(v_{r}(x)\right) d x \\
& \leq M\left|B_{r}\right|+\int_{B_{r}}\left|\nabla v_{r}(x)\right|^{2} d x
\end{aligned}
$$

hence

$$
\int_{B_{r}}|\nabla u(x)|^{2}-\left|\nabla v_{r}(x)\right|^{2} d x \leq C r^{n}
$$

On the other hand we have: 


$$
\begin{aligned}
\int_{B_{r}}|\nabla u(x)|^{2}-\left|\nabla v_{r}(x)\right|^{2} d x & =\int_{B_{r}}\left(\nabla u(x)+\nabla v_{r}(x), \nabla u(x)-\nabla v_{r}(x)\right) d x \\
& =\int_{B_{r}}\left|\nabla\left(u(x)-v_{r}(x)\right)\right|^{2} d x+2 \int_{B_{r}}\left(\nabla u-\nabla v_{r}\right) \nabla v_{r} d x \\
& =\int_{B_{r}}\left|\nabla\left(u(x)-v_{r}(x)\right)\right|^{2} d x
\end{aligned}
$$

where for the last inequality we used that $v_{r}$ is harmonic and equal to $u$ on $\partial B_{r}$.

Thus we get:

$$
\int_{B_{r}}\left|\nabla\left(u(x)-v_{r}(x)\right)\right|^{2} d x \leq C r^{n}
$$

From the previous estimate, it suffices to show that

$$
\int_{B_{s}}|\nabla u|^{2} \leq C s^{n}\left[\ln ^{2}(r / s)+1\right]
$$

This would imply (2.1).

To prove (2.7), we proceed as follows:

$$
\int_{B_{s}}|\nabla u|^{2} \leq \int_{B_{s}}\left|\nabla v_{2 s}\right|^{2}+\int_{B_{s}}\left|\nabla\left(u-v_{2 s}\right)\right|\left|\nabla\left(u+v_{2 s}\right)\right|
$$

The first integral on the right side is estimated using the subharmonicity of $\left|\nabla v_{2 s}\right|^{2}$, and then the minimality of $v_{2 s}$. So,

$$
\frac{1}{\left|B_{s}\right|} \int_{B_{s}}\left|\nabla v_{2 s}\right|^{2} \leq \frac{1}{\left|B_{2 s}\right|} \int_{B_{2 s}}\left|\nabla v_{2 s}\right|^{2} \leq \frac{1}{\left|B_{2 s}\right|} \int_{B_{2 s}}|\nabla u|^{2}
$$

by (2.5) $)$.

The second integral is estimated by enlarging the domain to $B_{2 s}$, then Cauchy-Schwartz, the established bound and the minimality of $v_{2 s}$

$$
\begin{gathered}
\frac{1}{\left|B_{s}\right|} \int_{B_{s}}\left|\nabla\left(u-v_{2 s}\right)\right|\left|\nabla\left(u+v_{2 s}\right)\right| \leq \\
\frac{\left|B_{2 s}\right|}{\left|B_{s}\right|}\left(\frac{1}{\left|B_{2 s}\right|} \int_{B_{2 s}} \mid \nabla\left(u-v_{2 s}\right)^{2}\right)^{\frac{1}{2}}\left(\frac{2}{\left|B_{2 s}\right|} \int_{B_{2 s}}|\nabla u|^{2}+\left|\nabla v_{2 s}\right|^{2}\right)^{\frac{1}{2}} \leq C\left(\frac{1}{\left|B_{2 s}\right|} \int_{B_{2 s}}|\nabla u|^{2}\right)^{\frac{1}{2}}
\end{gathered}
$$

by (2.5),$(2.6)$. 
So if we set

$$
x_{k}=\frac{1}{\left|B_{2^{-k}}\right|} \int_{B_{2^{-k}}}|\nabla u|^{2}
$$

then

$$
x_{k+1} \leq x_{k}+C x_{k}^{1 / 2}
$$

Induction gives

$$
x_{k+1} \leq C^{\prime} k^{2}
$$

from which you have (2.7).

Estimate (2.1) then follows from the proof of Morrey's embedding. Indeed, suppose $x$ and $y$ are given, of distance $2 s$ apart. Let $z$ be the midpoint. Then, by mean value theorem,

$$
\frac{1}{\left|B_{s}\right|} \int_{B_{s}}|u(x)-u(p)| d p \leq C s \frac{1}{\left|B_{s}\right|} \int_{B_{s}} \int_{0}^{1}|\nabla u(p+t(x-p))| d t d p
$$

Thus, interchanging the order of integration and using (2.7), we get

$$
\frac{1}{\left|B_{s}\right|} \int_{B_{s}}|u(x)-u(p)| d p \leq C s[\ln (1 / s)+1]
$$

The estimate for $|u(x)-u(y)|$ then follows from triangle inequality.

The proof for the case $\alpha=0$ is similar, the only difference being that instead of the bound in (2.2) $\left|W^{0}(u(x))\right|,\left|W^{0}\left(v_{r}(x)\right)\right| \leq 1$ is used.

\subsection{The Basic Estimate:}

Lemma 2.2. Let $u: \mathbb{R}^{n} \rightarrow \mathbb{R}^{m}$ minimizer of $J,|u(x)|<M, W$ satisfying (H1) for $0<\alpha<2$ and $W=W^{0}$ for $\alpha=0$. Then there is a constant $C_{0}=C_{0}(W, M)$ independent of $x_{0}$ and such that

$$
J_{B_{r}\left(x_{0}\right)}(u) \leq C_{0} r^{n-1} \quad, r>r_{0}
$$


Proof.

1. For $\alpha \in[1,2)$, utilizing elliptic estimates we obtain $|\nabla u(x)|<C(M), x \in \mathbb{R}^{n}$. The estimate then follows by constructing a competitor $v(x)$ on a ball via

$$
v(x)= \begin{cases}a \quad, & \left|x-x_{0}\right| \leq r-1 \\ \left(r-\left|x-x_{0}\right|\right) a+\left(\left|x-x_{0}\right|-r+1\right) u(x), r-1<\left|x-x_{0}\right| & \\ u(x) \quad, \quad\left|x-x_{0}\right|>r\end{cases}
$$

and utilizing the minimality of $u$ (cfr Lemma 5.1 [1]). Here we can take $r_{0}=0$.

2. For $\alpha \in(0,1)$, we aim to prove the estimate:

Lemma 2.3. Let $u: \mathbb{R}^{n} \rightarrow \mathbb{R}^{m}$ be a bounded local minimizer for the energy functional $J$ in (1.1) with the potential $W_{\alpha}$ as in (H1). Then there exists constant $C, R_{0}>0$ independent of $u$ such that:

$$
J(u ; A(R)) \leq C R^{n-1}, \forall R \geq R_{0}
$$

where $C$ is independent of $R \geq R_{0}$ and $A(R):=B_{R}\left(x_{0}\right) \backslash B_{R-1}\left(x_{0}\right)$.

Proof. We first claim that there exists a constant $\tilde{C}>0$ such that for any $x_{0} \in \mathbb{R}^{n}$ we have, for $u$ a bounded local minimizer:

$$
\int_{B_{1}\left(x_{0}\right)}|\nabla u(x)|^{2} d x \leq \tilde{C}
$$

To this end we consider the function $v \in W^{1,2}\left(B_{1}\left(x_{0}\right)\right)$ with $v=u$ on $\partial B_{1}\left(x_{0}\right)$ and $\Delta v=0$ in $B_{1}\left(x_{0}\right)$. Since $u$ is bounded, by the maximum principle we have that $v$ is also bounded and taking into account the hypothesis $(\mathbf{H 1})$ for the potential $W_{\alpha}$ we have that there exists $M>0$ such that:

$$
|u(x)|,|v(x)|, W_{\alpha}(u(x)), W_{\alpha}(v(x)) \leq M, \forall x \in \mathbb{R}^{n}, \alpha \in[0,1]
$$

We then have: 


$$
\begin{aligned}
& \int_{B_{1}\left(x_{0}\right)}|\nabla u(x)|^{2} d x \leq \int_{B_{1}\left(x_{0}\right)}|\nabla u(x)|^{2}+W_{\alpha}(u(x)) d x \leq \int_{B_{1}\left(x_{0}\right)}|\nabla v(x)|^{2}+W_{\alpha}(v(x)) d x \\
& \leq M\left|B_{1}\right|+\int_{B_{1}\left(x_{0}\right)}|\nabla v(x)|^{2} d x=M\left|B_{1}\right|+\int_{\partial B_{1}\left(x_{0}\right)} \frac{\partial v}{\partial \nu} v d \sigma \\
& \leq M\left|B_{1}\right|+\left\|\frac{\partial v}{\partial \nu}\right\|_{H^{-\frac{1}{2}\left(\partial B_{1}\left(x_{0}\right)\right)}}\|v\|_{H^{\frac{1}{2}\left(\partial B_{1}\left(x_{0}\right)\right)}} \\
& \leq M\left|B_{1}\right|+C\|\nabla v\|_{L^{2}\left(B_{1}\left(x_{0}\right)\right)}\|v\|_{H^{\frac{1}{2}\left(\partial B_{1}\left(x_{0}\right)\right)}} \\
& \leq M\left|B_{1}\right|+\frac{1}{2}\|\nabla v\|_{L^{2}\left(B_{1}\left(x_{0}\right)\right)}^{2}+C\|v\|_{H^{\frac{1}{2}\left(\partial B_{1}\left(x_{0}\right)\right)}}^{2} \\
& =M\left|B_{1}\right|+\frac{1}{2}\|\nabla v\|_{L^{2}\left(B_{1}\left(x_{0}\right)\right)}^{2}+C\|u\|_{H^{\frac{1}{2}\left(\partial B_{1}\left(x_{0}\right)\right)}}^{2}
\end{aligned}
$$

where for the first inequality we used the non-negativity of $W_{\alpha}$, for the second the local minimality of $u$, and for the third the estimates (2.10). For the first equality we used the fact that $v$ is a harmonic function and an integration by parts, while for the last equality we used that $u=v$ on $\partial B_{1}\left(x_{0}\right)$. For the penultimate inequality we used the continuity of the normal part of trace operator on the space $L_{d i v}^{2}=\left\{f \in L^{2}\right.$; div $\left.f \in L^{2}\right\}$ (see for instance Prop. 3.47, (ii) in [18]).

We obtain thus:

$$
\int_{B_{1}\left(x_{0}\right)}|\nabla u(x)|^{2} d x \leq M\left|B_{1}\right|+C\|u\|_{H^{\frac{1}{2}}\left(\partial B_{1}\left(x_{0}\right)\right)}^{2}
$$

On the other hand we have (see for instance [31]):

$$
\|u\|_{H^{\frac{1}{2}\left(\partial B_{1}\left(x_{0}\right)\right)}}^{2}=\int_{\partial B_{1}\left(x_{0}\right)} \int_{\partial B_{1}\left(x_{0}\right)} \frac{|u(x)-u(y)|^{2}}{|x-y|^{n-1+1}} d x d y \leq C
$$

where for the last inequality we used the logarithmic estimate (2.1).

Combining the last two estimates we obtain the claimed uniform estimate (2.9)). On the other hand, thanks to estimate (2.10) we have

$$
\int_{A(R)} W_{\alpha}(u(x)) d x \leq C R^{n-1}
$$

which combined with the fact that one can cover $A(R)$ with $C R^{n-1}$ balls of radius 1 and estimate (2.9) provides the desired estimate (2.8). 
Note: Lemma 2.3 implies Lemma $2.2(\alpha \in(0,1))$ by considering the comparison function $v(x)$ as in $\alpha \in[1,2)$ case.

\subsection{The "Dead Core" estimate:}

Now, we proceed with a useful calculation. From the hypothesis (H1) for $W$ we have that for $|u-a|<<1$, it holds that $W_{u}(u) \cdot(u-a) \geq \bar{c}^{2}|u-a|^{\alpha}$ with $\bar{c}^{2}=\alpha C^{*}, \alpha \in(0,2)$. Set $v(x)=|u-a|^{2}$.

Then

$$
\begin{gathered}
\Delta v=\sum_{i=1}^{n} 2\left((u(x)-a) u_{x_{i}}\right)_{x_{i}}=2|\nabla u|^{2}+2(u(x)-a) \Delta u= \\
2|\nabla u|^{2}+2 W_{u}(u) \cdot(u(x)-a) \geq 2|\nabla u|^{2}+2 \bar{c}^{2}|u-a|^{\alpha}
\end{gathered}
$$

Therefore,

$$
\Delta v \geq c^{2}|u-a|^{\alpha}=c^{2} v^{\frac{\alpha}{2}}, \text { where } c^{2}=2 \alpha C^{*}
$$

Definition 2.4. Let $\Omega \subset \mathbb{R}^{n}$ open and $v \in W_{\text {loc }}^{1,2}(\Omega, \mathbb{R})$, a region $\Omega_{0} \subset \Omega$ is called a dead core if $v \equiv 0$ in $\Omega_{0}$.

For the convenience of the reader, let us now state some results from [39].

The article [39] is concerned with the problem

$$
\left\{\begin{array}{l}
\Delta u=c^{2} u^{p} \text { in } \Omega \subset \mathbb{R}^{n} \\
u=1 \text { on } \partial \Omega
\end{array}\right.
$$

with $p \in(0,1)$. We call that a "dead core" $\Omega_{0}$ develops in $\Omega$, i.e. a region where $u \equiv 0$. 
Let $X(s)$ be a solution of

$$
\left\{\begin{array}{l}
X^{\prime \prime}(s)=c^{2} X^{p}(s) \text { in }\left(0, s_{0}\right) \\
X^{\prime}(0)=0, X\left(s_{0}\right)=1
\end{array}\right.
$$

As a first choice of a linear problem consider the "torsion problem", i.e.

$$
\left\{\begin{array}{l}
\Delta \psi+1=0 \text { in } \Omega \\
\psi=0 \text { on } \partial \Omega
\end{array}\right.
$$

One then constructs a supersolution $\bar{u}(x)$ to (2.17) having the same level lines as the torsion function by setting

$$
\bar{u}(x)=X(s(x)), \quad x \in \Omega
$$

where

$$
s(x)=\sqrt{2\left(\psi_{m}-\psi(x)\right)}, \psi_{m}=\max _{\Omega} \psi
$$

In problem (2.18) we choose $s_{0}=\sqrt{2 \psi_{m}}$.

Theorem 2. ([39]) Assume that the mean curvature of $\partial \Omega$ is nonnegative everywhere. Then

$$
\begin{gathered}
\bar{u}(x)=X(s(x)) \text { is a supersolution, i.e. } \\
\Delta \bar{u} \leq c^{2} \bar{u}^{p} \text { in } \Omega \\
\bar{u}=1 \text { on } \partial \Omega
\end{gathered}
$$

One of the corollaries of this Theorem is the information on the location and the size of the "dead core" $\Omega_{0}$, which may be stated as

Corollary 1. ([39]) The dead core $\Omega_{0}$ contains the set

$$
\left\{x \in \Omega \mid \psi(x) \geq d(p, c)\left[\sqrt{2 \psi_{m}}-\frac{1}{2} d(p, c)\right]\right\},
$$

where $d(p, c):=\frac{\sqrt{2(p+1)}}{(1-p) c}$. 
We will now utilize the above for the proof of the following Lemmas.

Lemma 2.5. Let $\Omega=B_{R}\left(x_{0}\right) \subset \mathbb{R}^{n}$ and $v \in C^{2}\left(\Omega ; \mathbb{R}_{+}\right)$satisfy the following assumptions:

$$
\begin{gathered}
\Delta v(x) \geq c^{2} v^{\frac{\alpha}{2}}(x), x \in \Omega \\
v(x) \leq \delta, x \in \partial \Omega
\end{gathered}
$$

$\alpha \in(0,2) \Leftrightarrow \frac{\alpha}{2}=p \in(0,1)$.

Then if $y_{0} \in \Omega$ is such that $\operatorname{dist}\left(y_{0}, \partial \Omega\right)>R_{0} \Rightarrow v\left(y_{0}\right)=0$.

where $R_{0}:=\left\{\begin{array}{l}\sqrt{n} d(p, \hat{c}), R \geq \sqrt{n} d(p, \hat{c}) \\ 2 R-\sqrt{n} d(p, \hat{c}), \frac{1}{2} \sqrt{n} d(p, \hat{c})<R<\sqrt{n} d(p, \hat{c})\end{array}\right.$

and $d(p, \hat{c}):=\frac{\sqrt{2(p+1)}}{(1-p) \hat{c}}, \hat{c}=\frac{c}{\delta^{\frac{1-p}{2}}}$.

Proof. From the maximum principle we have that $v(x) \leq \delta$ in $\Omega$

Define $\hat{v}:=\frac{v}{\delta}$ and $\hat{c}:=\frac{c}{\delta^{\frac{1-p}{2}}}$, then we have:

$$
\left\{\begin{array}{l}
\Delta \hat{v}(x) \geq \hat{c}^{2} \hat{v}^{\frac{\alpha}{2}}(x), x \in \Omega \\
\hat{v}(x) \leq 1, x \in \partial \Omega
\end{array}\right.
$$

For $\Omega=B_{R}\left(x_{0}\right)$ we have that

$$
\psi(x)=\frac{R^{2}}{2 n}-\frac{1}{2 n}\left|x-x_{0}\right|^{2}, \psi_{m}=\frac{R^{2}}{2 n}
$$

is a solution to the problem:

$$
\left\{\begin{array}{l}
\Delta \psi(x)+1=0, x \in \Omega \\
\psi(x)=0, x \in \partial \Omega
\end{array}\right.
$$

Also, we have that if:

$$
\left\{\begin{array}{l}
\Delta u \leq c^{2} u^{p}, x \in \Omega \\
\Delta v \geq c^{2} v^{p}, x \in \Omega \\
v \leq u, x \in \partial \Omega
\end{array}\right.
$$

then $v \leq u$, in $\Omega$. So since $u, v \geq 0$, if $u\left(x_{1}\right)=0 \Rightarrow v\left(x_{1}\right)=0$.

Such $u$ is defined in [39] via $\psi$ in Theorem 2] (supersolution with $u=1 \geq \hat{v}$ on the boundary). 
Then by Corollary 1 in [39], the dead core of $\bar{u}$ contains the set $\left\{x \in \Omega \mid \psi(x) \geq C_{0}:=\right.$ $\left.d(p, \hat{c})\left[\frac{R}{\sqrt{n}}-\frac{1}{2} d(p, \hat{c})\right]\right\}$, that is if $y_{0} \in\left\{\psi(x) \geq C_{0}\right\} \Rightarrow \bar{u}\left(y_{0}\right)=0$ and thus $\hat{v}\left(y_{0}\right)=v\left(y_{0}\right)=0$.

Since $\psi$ has the form (2.24) we can see that

$$
\left\{x \in \Omega \mid \psi(x) \geq C_{0}\right\}=\left\{\operatorname{dist}(x, \partial \Omega) \geq R_{0}\right\}
$$

as follows:

$$
\begin{gathered}
\psi(x) \geq C_{0} \Leftrightarrow \frac{R^{2}}{2 n}-\frac{1}{2 n}\left|x-x_{0}\right|^{2} \geq C_{0} \Leftrightarrow \sqrt{R^{2}-2 n C_{0}} \geq\left|x-x_{0}\right| \\
\Leftrightarrow R-\left|x-x_{0}\right| \geq R-\sqrt{R^{2}-2 n C_{0}}=R-\sqrt{R^{2}-2 \sqrt{n} d(p, \hat{c}) R+n(d(p, \hat{c}))^{2}}= \\
=R-|R-\sqrt{n} d(p, \hat{c})|=R_{0}
\end{gathered}
$$

and notice that: $\operatorname{dist}(x, \partial \Omega)=\operatorname{dist}\left(x, \partial B_{R}\left(x_{0}\right)\right)=R-\operatorname{dist}\left(x, x_{0}\right)$

Notes: (1) $\hat{c}$ depends on $\delta$ and tends to infinity as $\delta$ tends to zero.

(2) $d(p, \hat{c})$ tends to zero as $\delta$ tends to zero, and so does $C_{0}$.

Remark 2.6. If we take $\tilde{\Omega}$ open set, such that $B_{R}\left(x_{0}\right) \subset \tilde{\Omega}$ and

$$
\left\{\begin{array}{l}
\Delta \tilde{\psi}(x)+1=0, x \in \tilde{\Omega} \\
\tilde{\psi}(x)=0, x \in \partial \tilde{\Omega}
\end{array}\right.
$$

then, we have: $\psi \leq \tilde{\psi} \Rightarrow\left\{\psi(x) \geq C_{0}\right\} \subset\left\{\tilde{\psi}(x) \geq C_{0}\right\} \Rightarrow\left\{x \in B_{R}\left(x_{0}\right): \operatorname{dist}\left(\partial B_{R}\left(x_{0}\right), x\right) \geq\right.$ $\left.R_{0}\right\} \subset\left\{\tilde{\psi}(x) \geq C_{0}\right\}$.

Thus, the above theorem holds for more general open sets that contain a ball $B_{R}\left(x_{0}\right)$.

Lemma 2.7. Let $D$ open, convex $\subset \mathbb{R}^{n}$ and for some $d_{0}>0$, $\Omega:=\left\{x \in D: \operatorname{dist}(x, \partial D) \geq d_{0}\right\}$ and let $v \in C^{2}\left(D ; \mathbb{R}_{+}\right)$satisfying:

$$
\begin{gathered}
\Delta v(x) \geq c^{2} v^{\frac{\alpha}{2}}(x), x \in \Omega \\
v(x) \leq \delta, x \in \Omega
\end{gathered}
$$

$\alpha \in(0,2) \Leftrightarrow \frac{\alpha}{2}=p \in(0,1)$.

Then if $x_{0} \in D$ such that $\operatorname{dist}\left(x_{0}, \partial D\right) \geq d_{0}+2 \frac{\sqrt{2 n(p+1)}}{(1-p) \hat{c}} \Rightarrow v\left(x_{0}\right)=0$. 
Proof. We have that:

$$
\left\{x \in D: \operatorname{dist}(x, \partial D) \geq d_{0}+2 \frac{\sqrt{2 n(p+1)}}{(1-p) \hat{c}}\right\}=\left\{x \in \Omega: \operatorname{dist}(x, \partial \Omega) \geq 2 \frac{\sqrt{2 n(p+1)}}{(1-p) \hat{c}}\right\}
$$

and $\Omega$ is convex (parallel sets have at the same side of supporting planes).

Let $x_{0} \in D$ such that $\operatorname{dist}\left(x_{0}, \partial D\right) \geq d_{0}+2 \frac{\sqrt{2 n(p+1)}}{(1-p) \hat{c}}$. Since $\operatorname{dist}(\partial D, \partial \Omega)=d_{0} \Rightarrow \operatorname{dist}\left(x_{0}, \partial \Omega\right) \geq$ $2 \frac{\sqrt{2 n(p+1)}}{(1-p) \hat{c}}$ and since $\Omega$ is convex there exist a ball $B_{R}\left(x_{0}\right) \subset \Omega$ for $R=2 \frac{\sqrt{2 n(p+1)}}{(1-p) \hat{c}}=$ $2 \sqrt{n} d(p, \hat{c})>R_{0}=\sqrt{n} d(p, \hat{c}), d(p, \hat{c})$ as defined above.

Therefore we can apply Lemma 2.5 in the ball $B_{R}\left(x_{0}\right)$ and we have that $v(x)=0, \forall x \in$ $B_{R_{0}}\left(x_{0}\right)=\left\{x \in B_{R}\left(x_{0}\right): \operatorname{dist}\left(\partial B_{R}\left(x_{0}\right), x\right) \geq R_{0}\right\} \Rightarrow v\left(x_{0}\right)=0$.

The results of Lemma 2.5 and Lemma 2.7 above were proved for the case $1<\alpha<2$, since $u \in C^{2, \alpha-1}$ by elliptic regularity. However, they also hold for the case where $0<\alpha \leq 1$. The only difference in proving this, is that the differential inequality (2.16) holds weakly and we utilize it together with the weak maximum principle for the comparison argument as in the proof of lemma 2.5. So in order to extend the results of the lemmas above for the case where $0<\alpha \leq 1$, it suffices to prove the following claim.

\section{Lemma 2.8 .}

$$
\Delta v \geq c^{2} v^{\frac{\alpha}{2}} \quad \text { weakly in } W^{1,2}\left(B_{R}\left(x_{0}\right)\right) .
$$

\section{Proof.}

Let $v \in W^{1,2}\left(B_{R}\left(x_{0}\right)\right), v$ continuous $\left(v=|u-a|^{2}\right.$, by Lemma 2.1) and $v \geq 0$. We define $v_{\varepsilon}:=\max \{v, \varepsilon\}, 0<\varepsilon<\delta$ (where $\delta$ as in the above Lemmas). The set $\{v=\varepsilon\}$ is smooth by Sard's theorem, since $v$ is smooth away from zero.

Let $\phi \in C_{0}^{1}\left(B_{R}\left(x_{0}\right)\right), B_{R}^{\varepsilon}\left(x_{0}\right)=\{v>\varepsilon\} \cap B_{R}\left(x_{0}\right)$, we have 


$$
\begin{gathered}
-\int_{B_{R}\left(x_{0}\right)} \nabla v \nabla \phi d x=\lim _{\varepsilon \rightarrow 0} \int_{B_{R}^{\varepsilon}\left(x_{0}\right)}-\nabla v_{\varepsilon} \nabla \phi d x=\liminf _{\varepsilon \rightarrow 0}\left[-\int_{B_{R}^{\varepsilon}\left(x_{0}\right)} \nabla v \nabla \phi d x\right] \\
\geq \liminf _{\varepsilon \rightarrow 0}\left[\int_{B_{R}^{\varepsilon}\left(x_{0}\right)} \Delta v \phi d x-\int_{\partial B_{R}^{\varepsilon}\left(x_{0}\right)} \frac{\partial v}{\partial \nu} \phi d S\right] \geq \liminf _{\varepsilon \rightarrow 0}\left[\int_{B_{R}^{\varepsilon}\left(x_{0}\right)} \Delta v \phi d x\right] \\
\geq \liminf _{\varepsilon \rightarrow 0}\left[\int_{B_{R}^{\varepsilon}\left(x_{0}\right)} c^{2} v^{\frac{\alpha}{2}} \phi d x\right]=\lim _{\varepsilon \rightarrow 0}\left[\int_{B_{R}^{\varepsilon}\left(x_{0}\right)} c^{2} v^{\frac{\alpha}{2}} \phi d x\right]= \\
\geq \lim _{\varepsilon \rightarrow 0}\left[\int_{B_{R}\left(x_{0}\right)} c^{2} v_{\varepsilon}^{\frac{\alpha}{2}} \phi d x-c^{2} \varepsilon^{\frac{\alpha}{2}} \int_{B_{R} \backslash B_{R}^{\varepsilon}} \phi d x\right]=\int_{B_{R}\left(x_{0}\right)} c^{2} v^{\frac{\alpha}{2}} \phi d x .
\end{gathered}
$$

\subsection{On the definition of $W^{0}$}

In what follows we establish essentially that $\lim _{\alpha \rightarrow 0} J^{\alpha}=J^{0}$ in the $\Gamma$ - convergence sense. The containment result in Appendix $\mathrm{A}$ is essential here.

$$
J^{\alpha}(\Omega, u)=\int_{\Omega}\left(\frac{1}{2}|\nabla u|^{2}+W^{\alpha}(u)\right) d x
$$

with

$$
W^{\alpha}(u):=\prod_{i=1}^{N}\left|u-a_{i}\right|^{\alpha}, i \in\{1, \ldots, N\} 0<\alpha<2 .
$$

We further denote:

$$
W_{0}(u):=\chi_{\left\{u \in S_{A}\right\}}
$$

where

$$
A:=\left\{a_{1}, \ldots, a_{N}\right\}
$$

and 


$$
S_{A}:=\left\{\sum_{i=1}^{N} \lambda_{i} a_{i}, \text { where } \sum_{i=1}^{N} \lambda_{i}=1, \lambda_{i} \in[0,1), i \in\{1, \ldots, N\}\right\}
$$

(i.e. $S_{A}$ is the convex hull of the points in $A$ except the point themselves). Then

$$
\bar{S}_{A}=S_{A} \cup A
$$

We have the following:

Lemma 2.9. Let $\left(u^{\alpha_{k}}\right)_{k \in \mathbb{N}}$ be a sequence of functions such that $\alpha_{k} \rightarrow 0$ as $k \rightarrow \infty$ and for any $k \in \mathbb{N}$ the function $u^{\alpha_{k}}: \mathbb{R}^{n} \rightarrow \mathbb{R}^{m}$ is an energy minimizer of $J^{\alpha_{k}}$ as defined in (2.28).

We assume that

$$
u^{\alpha_{k}}(x) \in \bar{S}_{A}, \forall x \in \mathbb{R}^{n}, k \in \mathbb{N}
$$

Then there exists a subsequence relabelled for simplicity as the initial sequence such that:

$$
u^{\alpha_{k}} \rightarrow \tilde{u}, \text { in } W^{1,2}\left(\mathbb{R}^{n} ; \mathbb{R}^{m}\right) \text {, as } k \rightarrow \infty
$$

with $\tilde{u}$ a local energy minimizer of the functional $J^{0}$ defined as:

$$
J^{0}(\Omega, u):=\int_{\Omega} \frac{1}{2}|\nabla u|^{2}+W^{0}(u(x)) d x
$$

(with $W^{0}$ from (2.30) ).

Proof. We have

$$
(P)\left\{\begin{array}{l}
W^{\alpha_{k}}(u) \rightarrow W^{0}(u) \quad \text { in } \bar{S}_{A} \text { as } k \rightarrow \infty \\
W^{\alpha_{k}} \geq 0, \forall \alpha_{k}>0
\end{array}\right.
$$

Arguing along the lines of Lemma 2.3, (while taking into account the properties $(P)$ and the definition (2.29) of $W^{\alpha_{k}} \mathrm{~s}$ ) we get:

$$
J^{\alpha_{k}}\left(B_{r}, u^{\alpha_{k}}\right) \leq C r^{n-1}
$$


for all $r \geq 1$, where $C$ depends only on the points $a_{1}, \ldots, a_{N}$ through the assumed inclusion (2.32) (and is independent of $\alpha_{k}, k \in \mathbb{N}$ ).

Out of this uniform bound we claim that there exists $\tilde{u} \in W^{1,2}\left(\mathbb{R}^{n} ; \mathbb{R}^{m}\right)$ such that:

(1) $u^{\alpha_{k}} \rightarrow \tilde{u}$ in $W^{1,2}\left(\mathbb{R}^{n} ; \mathbb{R}^{m}\right)$ as $k \rightarrow \infty$ on a subsequence

(2) $\tilde{u}$ is a local minimizer of $J^{0}$.

By the bound (2.35),$W^{\varepsilon} \geq 0$ and by the Rellich- Kondrachov theorem, we can obtain, along a subsequence

$$
u^{\alpha_{k}} \rightarrow \tilde{u} \quad \text { on } W^{1,2}\left(\mathbb{R}^{n} ; \mathbb{R}^{m}\right)
$$

and

$$
u^{\alpha_{k}} \rightarrow \tilde{u} \quad \text { on } L_{l o c}^{p}\left(\mathbb{R}^{n} ; \mathbb{R}^{m}\right)
$$

These provide claim (1).

In order to show claim (2) we note first we have:

$$
J^{0}(\tilde{u}, \Omega) \leq \liminf _{\alpha_{k} \rightarrow 0} J^{\alpha_{k}}\left(u^{\alpha_{k}}, \Omega\right)
$$

Indeed, we have by lower semicontinuity

$$
\int_{\Omega}|\nabla \tilde{u}|^{2} d x \leq \liminf _{k \rightarrow \infty} \int_{\Omega}\left|\nabla u^{\alpha_{k}}\right|^{2} d x
$$

We have that $\tilde{u} \in \bar{S}_{A}$ and we denote $A_{\tilde{u}}:=\left\{x \in \mathbb{R}^{n}: \tilde{u}(x) \in S_{A}\right\}$. Taking into account the specific form (2.29) of the potential $W^{\alpha}$ we have, for $\alpha_{k} \rightarrow 0$ as $k \rightarrow \infty$ :

$$
\int_{A_{\tilde{u}} \cap \Omega} \chi_{\left\{\tilde{u} \in S_{A}\right\}} d x=\int_{A_{\tilde{u}} \cap \Omega} d x=\lim _{k \rightarrow \infty} \int_{A_{\tilde{u} \cap \Omega}} W_{\alpha_{k}}\left(u^{\alpha_{k}}(x)\right) d x
$$

Furthermore, since $W^{\alpha} \geq 0$ we have:

$$
\int_{\Omega \backslash A_{\tilde{u}}} \chi_{\left\{\tilde{u} \in S_{A}\right\}} d x=0 \leq \lim _{k \rightarrow \infty} \int_{\Omega \backslash A_{\tilde{u}}} W^{\alpha_{k}}\left(u^{\alpha_{k}}(x)\right) d x
$$

The last three estimates provide the claimed relation (2.36). One can then trivially see that:

$$
\inf J^{0}(\cdot, \Omega) \leq J^{0}(\tilde{u}, \Omega) \leq \liminf _{\alpha_{k} \rightarrow 0} \inf J^{\alpha_{k}}(\cdot, \Omega)
$$


We claim now that for an arbitrary $u \in W_{l o c}^{1,2}\left(\mathbb{R}^{n} ; \mathbb{R}^{m}\right)$ with $u(x) \in \bar{S}_{A}$ for almost all $x \in \mathbb{R}^{n}$ we have:

$$
\lim _{\alpha_{k} \rightarrow 0} J^{\alpha_{k}}(u, \Omega)=J^{0}(u, \Omega)
$$

Indeed we have:

$$
\begin{gathered}
\int_{A_{u} \cap \Omega} \chi_{\left\{u \in S_{A}\right\}} d x=\int_{A_{u} \cap \Omega} d x=\lim _{k \rightarrow \infty} \int_{A_{u} \cap \Omega} W^{\alpha_{k}}(u(x)) d x \\
\int_{\Omega \backslash A_{u}} \chi_{\left\{u \in S_{A}\right\}} d x=0=\lim _{k \rightarrow \infty} \int_{\Omega \backslash A_{u}} W^{\alpha_{k}}(u(x)) d x
\end{gathered}
$$

SO

$$
\int_{\Omega}|\nabla u|^{2}+\chi_{\left\{u \in S_{A}\right\}} d x=\lim _{k \rightarrow \infty} \int_{\Omega}|\nabla u|^{2}+W^{\alpha_{k}}(u(x)) d x
$$

as claimed.

We note now that (2.41) implies:

$$
J^{0}(u, \Omega)=\lim _{\alpha_{k} \rightarrow 0} J^{\alpha_{k}}(u, \Omega)=\limsup _{\alpha_{k} \rightarrow 0} J^{\alpha_{k}}(u, \Omega) \geq \limsup _{\alpha_{k} \rightarrow 0} \inf J^{\alpha_{k}}(\cdot, \Omega)
$$

and since this holds for $u$ arbitrary we get:

$$
\inf J^{0}(\cdot, \Omega) \geq \limsup _{\alpha_{k} \rightarrow 0} \inf J^{\alpha_{k}}(\cdot, \Omega)
$$

The last inequality, together with (2.40) provide the claimed local minimality of $\tilde{u}$.

Note: The above Lemma also holds for the class of local minimizers of the energy. 


\section{Proofs}

\subsection{Proof of Proposition 1}

Proof. (i) (cfr [1] p.161). Let

$$
|u(x)-a|<M, \quad\|u\|_{C^{\beta}}<\hat{C}=\hat{C}(M), x \in \mathcal{O}
$$

where for the Hölder bound we utilized Lemma 2.1. Given $q \in(0, M)$, assume that

$$
\left|u\left(x_{0}\right)-a\right| \geq q
$$

Then the Hölder continuity of $u$ implies that the hypothesis of the Density Estimate (1.24) is satisfied for

$$
\lambda=\frac{q}{2}, r_{0}=\left(\frac{q / 2}{\hat{C}}\right)^{\frac{1}{\beta}}, \mu_{0}=\mathcal{L}^{n}\left(B_{r_{0}}\left(x_{0}\right)\right)
$$

Therefore

$$
\mathcal{L}^{n}\left(B_{r}\left(x_{0}\right) \cap\left\{|u-a|>\frac{q}{2}\right\}\right) \geq C r^{n} \quad, B_{r}\left(x_{0}\right) \subset \mathcal{O}, r \geq r_{0}
$$

Let

$$
0<w_{\frac{q}{2}}:=\min _{\Sigma} W(z), \Sigma=\left\{|z-a|>\frac{q}{2}\right\} \cap\{d(z,\{W=0\} \backslash a) \geq k\}
$$

From this and the Basic Estimate Lemma 2.2 we obtain

$$
w_{\frac{q}{2}} C_{1} r^{n} \leq J_{B_{r}\left(x_{0}\right)}(u) \leq C_{0} r^{n-1}
$$

which is impossible for

$$
r>\frac{C_{0}}{w_{\frac{q}{2}} C_{1}}
$$

Therefore if we set

$$
r_{q}=\frac{2 C_{0}}{w_{\frac{q}{2}} C_{1}}
$$

then $B_{r_{q}}\left(x_{0}\right) \subset \mathcal{O}$ is incompatible with (3.2).

The proof of (i) is complete. 
(ii) Consider the ball $B_{R}\left(x_{0}\right), R$ to be selected.

Let $\xi \in B_{R}\left(x_{0}\right)$

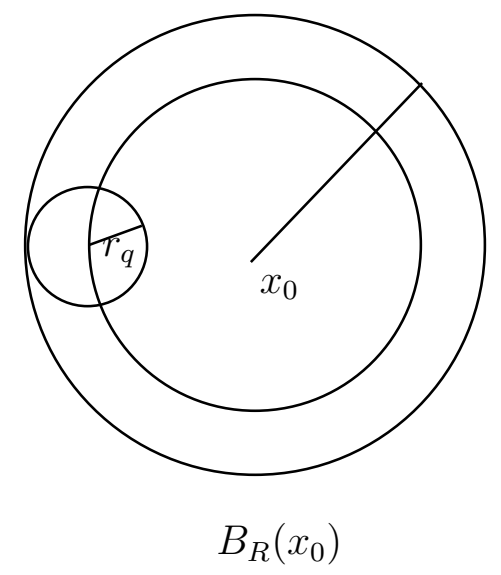

$$
d\left(\xi, \partial B_{R}\left(x_{0}\right)\right)=r_{q}, 0<2 q<\rho_{0}
$$

where $r_{q}$ as in (i) above. Note that by (H1)

$$
w_{\frac{q}{2}} \geq C^{*}\left(\frac{q}{2}\right)^{\alpha}, r_{q}=\frac{2 C_{0}}{w_{\frac{q}{2}} C_{1}} \leq \frac{2 C_{0}}{C_{1} C^{*}}\left(\frac{q}{2}\right)^{-\alpha}
$$

and by (i) above

$$
|u(\xi)-a|<q
$$

Therefore by [1], Theorem 4.1 originally derived in [5]

$$
|u(x)-a|<q, x \in B_{R-r_{q}}\left(x_{0}\right)
$$

By (2.16) $v(x):=|u(x)-a|^{2}$ satisfies

$$
\left\{\begin{array}{l}
\Delta v \geq c^{2} v^{\frac{\alpha}{2}} \text { weakly in } W^{1,2}\left(B_{R-r_{q}}\left(x_{0}\right)\right) \\
v \leq \delta \quad \text { on } \partial B_{R-r_{q}}\left(x_{0}\right)
\end{array}\right.
$$

and therefore by Lemma 2.5

$$
d\left(y_{0}, \partial B_{R-r_{q}}\left(x_{0}\right)\right)>R_{0} \Rightarrow v\left(y_{0}\right)=0
$$

where

$$
R_{0}=\frac{\sqrt{n(\alpha+2)}}{\left(1-\frac{\alpha}{2}\right) c} q^{1-\frac{\alpha}{2}}, 0<\alpha<2, c^{2}=2 \alpha C^{*}
$$


Therefore

$$
u(x)=a \text { in } B_{R-r_{q}-R_{0}}\left(x_{0}\right)
$$

To conclude set $R=C q^{-\alpha}$ and impose the requirement that

$$
\frac{C}{2} q^{-\alpha} \leq C q^{-\alpha}-r_{q}-R_{0}
$$

which is satisfied if

$$
C \geq \frac{2^{\alpha+2} C_{0}}{C_{1} C^{*}}+2 \frac{\sqrt{n(\alpha+2)}}{\left(1-\frac{\alpha}{2}\right) \sqrt{2 \alpha C^{*}}}\left(\frac{\rho_{0}}{2}\right)^{1+\frac{\alpha}{2}}=: \hat{C}(\alpha, n)
$$

The proof of Proposition 1 is complete.

\subsection{Proof of Theorem 1}

Proof. Step 1 (Existence of a positive minimizer)

We will be establishing the existence of a map $u_{R} \in W^{1,2}\left(B_{R}, \mathbb{R}^{n}\right)$ that is equivariant, positive and also a minimizer in the equivariant class of

$$
J_{B_{R}}(u)=\int_{B_{R}}\left(\frac{1}{2}|\nabla u|^{2}+W(u)\right) d x, B_{R}=\{|x|<R\} \subset \mathbb{R}^{n},
$$

that satisfies the Basic Estimate

$$
J_{B_{r}}\left(u_{R}\right) \leq C r^{n-1}, r_{0}<r<R, R \geq R_{0}
$$

$C$ independent of $R, r$.

We introduce the regularized energy functional

$$
J_{B_{R}}^{\varepsilon}(u)=\int_{B_{R}}\left(\frac{1}{2}|\nabla u|^{2}+W^{\varepsilon}(u)\right) d x
$$

where $W^{\varepsilon}$ is obtained from $W$ by regularizing only at the minima as in Figure below. 


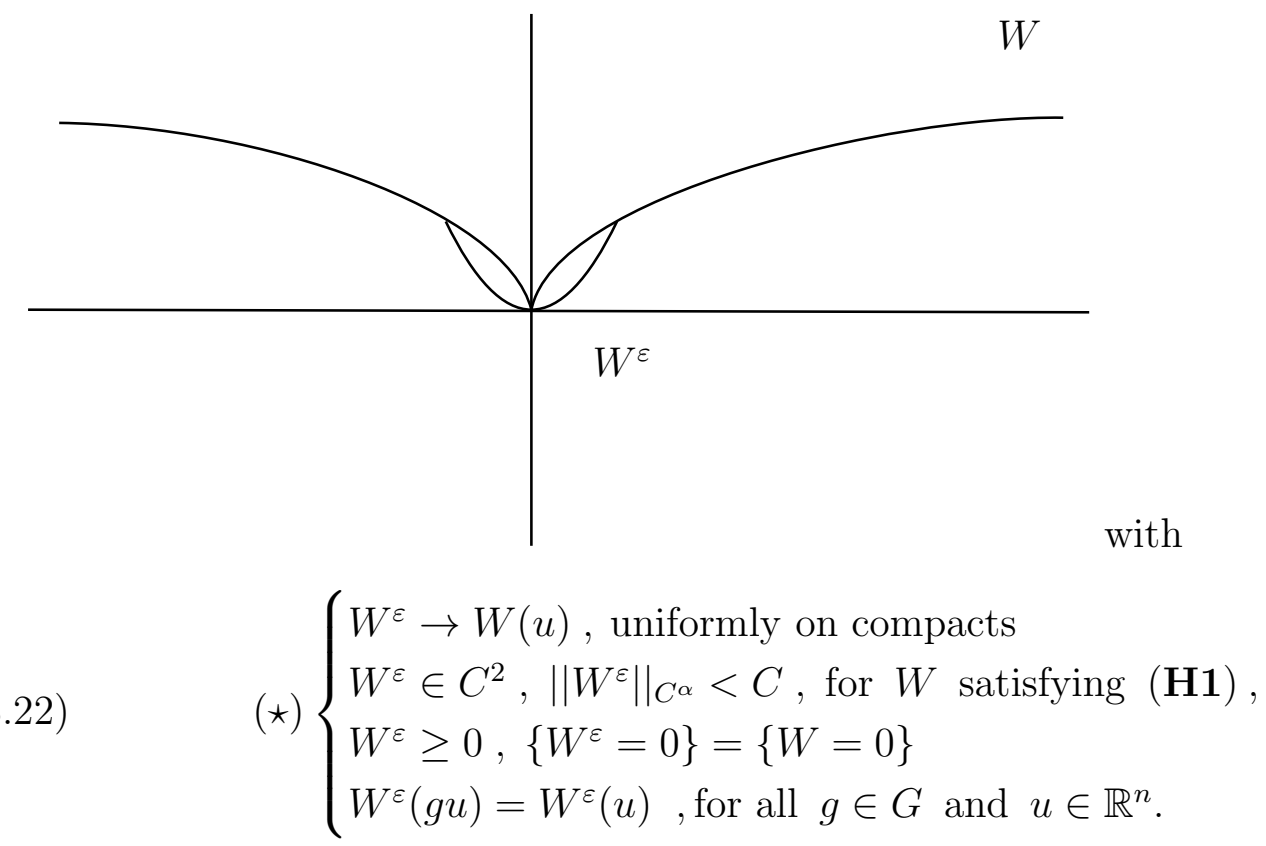

We can assume that

$$
W^{\varepsilon}(u)=W(u) \text { for }|u| \geq M>0
$$

some $M>0$, and that the minimizer of $J_{B_{R}}^{\varepsilon}$ in the equivariant class satisfies the bound

$$
\left|u_{R}^{\varepsilon}\right| \leq M \quad, x \in B_{R}
$$

with $M$ independent of $\varepsilon$ and $R$ and that moreover $u_{R}^{\varepsilon}$ is positive. Here we are utilizing [1] Lemma 6.1.

We begin by establishing the Hölder Estimate (1.25), for $u_{R}^{\varepsilon}$, with constant $C$ independent of $\varepsilon, R$. Recall that $u_{R}^{\varepsilon}$ is a minimizer in the equivariant class, while (1.25) was derived under the stronger hypothesis of being a minimizer under arbitrary perturbations. We point out only the necessary modifications of the proof of the Lemma 2.1.

We will derive

$$
\left|u_{R}^{\varepsilon}(x)-u_{R}^{\varepsilon}(y)\right| \leq C|x-y| \ln |x-y|^{-1} \quad, \forall x, y \in B_{R}(0) \backslash B_{1}(0)
$$

with $|x-y| \leq \frac{1}{2}, R \geq 2$.

Notice that we can cover $F_{R} \cap\left(B_{R}(0) \backslash B_{1}(0)\right)=: F_{R, D}$ where $F_{R}=F \cap B_{R}(0)$ by two types of balls $B_{\frac{1}{4}}\left(x_{0}\right)$ :

(a) Balls entirely contained in $F_{R, D}, B_{\frac{1}{4}} \subset F_{R, D}$,

(b) balls $B_{\frac{1}{4}}\left(x_{0}\right)$ having their center in the wall of $F_{R}$ which is made up of reflection planes 
in $G_{a}$.

Notice that both types can be equivariantly extended over $B_{R}(0) \backslash B_{1}(0)$ as sets.

Fix now $B_{r}\left(x_{0}\right), r<\frac{1}{4}$ as in the proof of (2.1). Due to the equivariant extension of $v_{r}$ there, and the minimality of $u_{R}^{\varepsilon}$ in the equivariant class, we see that $u_{R}^{\varepsilon}$ has the minimizing property on $B_{r}\left(x_{0}\right)$ and so (2.3) applies as before. The rest of the argument is unchanged.

Thus (3.25) is established.

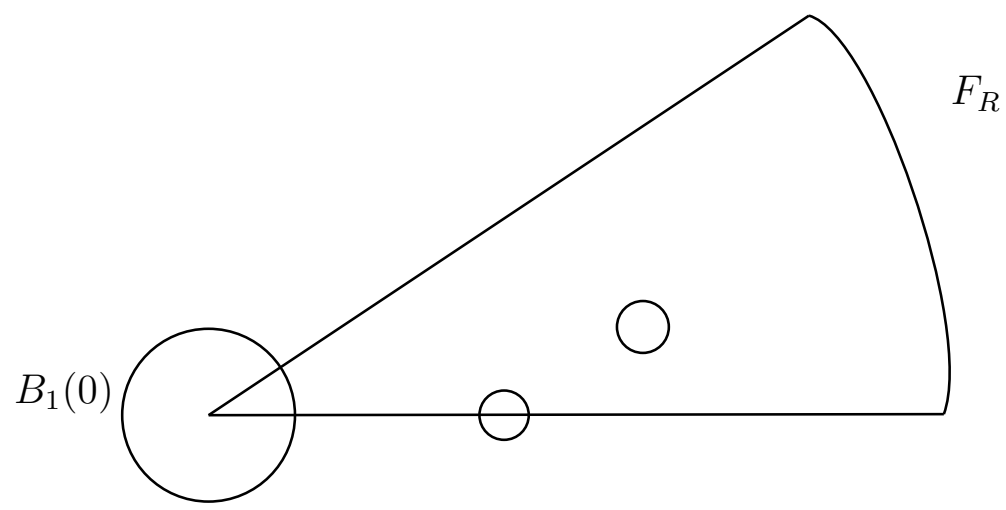

Fig :Typical $B_{\frac{1}{4}}\left(x_{0}\right)^{\prime} s$ covering the fundamental region and extensible equivariantly on $B_{R}(0) \backslash B_{1}(0)$.

Now we will proceed to establish (3.20),

$$
J_{B_{r}(0)}\left(u_{R}^{\varepsilon}\right) \leq C r^{n-1} \quad, \forall r \in(2, R-1)
$$

with $C$ constant independent of $\varepsilon$ and $R, C=C(M)$.

We follow [1] Proposition 6.1, and for $2<r<R-1$ we define

$$
u_{a f f}(x)= \begin{cases}d(x, \partial D) a_{1} & , \text { for } x \in D_{R} \text { and } d(x, \partial D) \leq 1 \\ a_{1} & , \text { for } x \in D_{R} \text { and } d(x, \partial D) \geq 1\end{cases}
$$

where $D_{R}=D \cap B_{R}$ and extend equivariantly in $B_{R}$. Since $u_{a f f}$ vanishes on $\partial D$, the extended map is also continuous. As it is well known, the distance is 1-Lipschitz and therefore in $W^{1, \infty}\left(B_{R}\right)$. Fix now a number $h \in(0,1)$ and for $r \in(2, R-1)$ define

$$
\hat{u}_{R}^{\varepsilon}(x)=\varphi\left(1-\frac{|x|-(r-h)}{h}\right) u_{a f f}(x)+\phi\left(\frac{|x|-(r-h)}{h}\right) u_{R}(x)
$$

where $\phi: \mathbb{R} \rightarrow[0,1]$ is a fixed $C^{1}$ function such that $\phi(s)=0$, for $s \leq 0$ and $\phi(s)=1$, for $s \geq 1$. Note that $\hat{u}_{R}^{\varepsilon} \in W_{E}^{1,2}\left(B_{R}(0) ; \mathbb{R}^{n}\right.$ ) (equivariant), and most importantly $\hat{u}_{R}^{\varepsilon}=u_{R}^{\varepsilon}$ on 
$\partial B_{r}(0)$. Moreover $\hat{u}_{R}=u_{a f f}$ in $B_{r-h}(0)$ and $\hat{u}_{R}^{\varepsilon}=u_{R}^{\varepsilon}$ on $B_{R}(0) \backslash B_{1}(0)$ and $u_{a f f}=a_{1}$ if $d(x, \partial D) \geq 1$. By the minimality of $u_{R}^{\varepsilon}$ we have

$$
\begin{gathered}
J_{B_{r}(0)}\left(u_{R}^{\varepsilon}\right) \leq J_{B_{r}(0)}\left(\hat{u}_{R}^{\varepsilon}\right) \\
=\int_{B_{r-h} \cap\{d(x, \partial D) \leq 1\}}\left(\frac{1}{2}\left|\nabla \hat{u}_{R}^{\varepsilon}\right|^{2}+W\left(\hat{u}_{R}^{\varepsilon}\right)\right) d x+\int_{B_{r} \backslash B_{r-h}}\left(\frac{1}{2}\left|\nabla \hat{u}_{R}^{\varepsilon}\right|^{2}+W\left(\hat{u}_{R}^{\varepsilon}\right)\right) d x \\
\leq C_{1}(r-h)^{n-1}+C_{2} r^{n-1}
\end{gathered}
$$

where for the estimate of the $2^{\text {nd }}$ term we used the Hölder estimate above and the analogous (2.12), (2.13).

Hence (3.26) is established.

Thus for any $R>0$ there exists $C_{R}>0$, independent of $\varepsilon>0$, such that

$$
\int_{B_{R}}\left(\frac{1}{2}\left|\nabla u_{R}^{\varepsilon}\right|^{2}+W^{\varepsilon}\left(u_{R}^{\varepsilon}\right)\right) d x<C_{R}
$$

Out of the above uniform bounds we claim that there exists $u_{R} \in W^{1,2}\left(B_{R} ; \mathbb{R}^{m}\right)$ such that

(1) $u_{R}^{\varepsilon} \rightarrow u_{R}$ weakly in $W^{1,2}\left(B_{R} ; \mathbb{R}^{m}\right)$ as $\varepsilon \rightarrow 0$ on a subsequence,

(2) $u_{R}$ is a minimizer of

$$
J_{B_{R}}(u)=\int_{B_{R}}\left(\frac{1}{2}|\nabla u|^{2}+W(u)\right) d x
$$

(3) $J_{B_{r}}\left(u_{R}\right) \leq C r^{n-1}$ with $C$ independent of $\varepsilon$ and $R$,

(4) $u_{R}$ is equivariant and positive.

By (3.30) and $W^{\varepsilon} \geq 0$ and the Rellich-Kondrachov theorem, we can obtain, for a subsequence

$$
u_{R}^{\varepsilon} \rightarrow u_{R} \text { on } W^{1,2}\left(B_{R} ; \mathbb{R}^{m}\right)
$$

and

$$
u_{R}^{\varepsilon} \rightarrow u_{R} \quad \text { on } \quad L^{p}\left(B_{R} ; \mathbb{R}^{m}\right)
$$

These establish claims (1) and (4). 
In order to show claim (2) we take $\phi \in C_{c}^{\infty}\left(\mathbb{R}^{n}\right), \operatorname{supp} \phi \subset K \subset B_{R}$. Then by minimality we have:

$$
\begin{gathered}
J_{B_{R}}^{\varepsilon}\left(u_{R}^{\varepsilon}+\phi\right)-J_{B_{R}}^{\varepsilon}\left(u_{R}^{\varepsilon}\right) \geq 0 \\
\Leftrightarrow \int_{B_{R}}\left(\nabla u_{R}^{\varepsilon} \nabla \phi+\frac{1}{2}|\nabla \phi|^{2}+W^{\varepsilon}\left(u_{R}^{\varepsilon}+\phi\right)-W^{\varepsilon}\left(u_{R}^{\varepsilon}\right)\right) d x \geq 0
\end{gathered}
$$

Let $I_{1}^{\varepsilon}:=\int_{B_{R}} \nabla u_{R}^{\varepsilon} \nabla \phi d x$ and $I_{2}^{\varepsilon}:=\int_{B_{R}}\left(W^{\varepsilon}\left(u_{R}^{\varepsilon}+\phi\right)-W^{\varepsilon}\left(u_{R}^{\varepsilon}\right)\right) d x$.

Thanks to (1) before we have $I_{1}^{\varepsilon} \rightarrow I_{1}=\int_{B_{R}} \nabla u_{R} \nabla \phi d x$ we split:

$$
I_{2}=\int_{B_{R}}\left(W^{\varepsilon}\left(u_{R}^{\varepsilon}+\phi\right)-W\left(u_{R}^{\varepsilon}+\phi\right)\right) d x+\int_{B_{R}}\left(W\left(u_{R}^{\varepsilon}+\phi\right)-W^{\varepsilon}\left(u_{R}^{\varepsilon}\right)\right) d x
$$

Let $I_{21}^{\varepsilon}:=\int_{B_{R}}\left(W^{\varepsilon}\left(u_{R}^{\varepsilon}+\phi\right)-W\left(u_{R}^{\varepsilon}+\phi\right)\right) d x$ and $I_{22}^{\varepsilon}:=\int_{B_{R}}\left(W\left(u_{R}^{\varepsilon}+\phi\right)-W^{\varepsilon}\left(u_{R}^{\varepsilon}\right)\right) d x \quad, I_{21}^{\varepsilon} \rightarrow 0$ as $\varepsilon \rightarrow 0$ because of the uniform bound $\left|u_{R}^{\varepsilon}(x)\right| \leq M$ the uniform convergence on compacts of $W^{\varepsilon}$ to $W$ and the dominated convergence theorem.

Also $I_{22}^{\varepsilon} \rightarrow I_{22}=\int_{B_{R}}\left(W\left(u_{R}+\phi\right)-W\left(u_{R}\right)\right) d x$ because of the $L^{p}$ convergence of $u_{R}^{\varepsilon}$ to $u_{R}$, dominated convergence and continuity of $W$.

Thus we establish the claimed relation (2).

In order to get the claimed relation (3) we recall

$$
J_{B_{r}}^{\varepsilon}\left(u_{R}^{\varepsilon}\right)=\int_{B_{r}}\left(\frac{1}{2}\left|\nabla u_{R}^{\varepsilon}\right|^{2}+W^{\varepsilon}\left(u_{R}^{\varepsilon}\right)\right) d x \leq C r^{n-1}
$$

with $C$ depending only on $M$, but not on $R$ nor on $\varepsilon$.

As $u_{R}^{\varepsilon} \rightarrow u_{R}$ in $W^{1,2} \Rightarrow \int_{B_{R}}\left|\nabla u_{R}\right|^{2} d x \leq \liminf \int_{B_{R}} \frac{1}{2}\left|\nabla u_{R}^{\varepsilon}\right|^{2} d x$ and we have

$$
\int_{B_{R}} W^{\varepsilon}\left(u_{R}^{\varepsilon}\right) d x \rightarrow \int_{B_{R}} W\left(u_{R}\right) d x
$$

arguing as in the treatment of the $I_{2}$ before.

Claim: There exists $\bar{u} \in W_{l o c}^{1,2}\left(\mathbb{R}^{n} ; \mathbb{R}^{m}\right)$ nontrivial equivariant, positive and minimizer of

$$
J_{\Omega}(u)=\int_{\Omega}\left(\frac{1}{2}|\nabla u|^{2}+W(u)\right) d x
$$

In addition, $\bar{u}$ satisfies the estimate

$$
J_{B_{r}}(\bar{u}) \leq c r^{n-1}
$$




\section{Proof.}

We have that out of the uniform bound $J_{B_{r}}\left(u_{R}\right) \leq c r^{n-1}$, we get as before, in the proof of the claims (1)-(4) that $u_{R} \rightarrow \bar{u}$ in $W_{l o c}^{1,2}\left(\mathbb{R}^{n} ; \mathbb{R}^{m}\right)$ and that $\bar{u}$ is equivariant and positive. We can argue similarly as in the proof of (2) above to get that $\bar{u}$ is a minimizer of $J_{\Omega}$ defined in (3.31), (3.32) follows from (3).

Step 2. (Existence of a free boundary)

We utilize that $D$ contains a unique zero $a_{1}$ of $W$ and that by equivariance we can restrict $u$ in $D$ and note that

$$
d\left(u(D),\{W=0\} \backslash a_{1}\right) \geq k>0
$$

For implementing Proposition 1 we need a couple of observarions. Firstly $u$ is minimizing in the class of equivariant positive maps. We recall that in the proof of Proposition 1 the density estimate (1.24) is utilized. We note that in the proof of the density estimate the energy comparison maps are obtained by reducing the modulus of the map $q^{u}(x)=\left|u(x)-a_{1}\right|$ and leaving the angular part $\nu^{u}(x)$ unchanged, $u(x)=a_{1}+q^{u}(x) \nu^{u}(x), \sigma(x)=a_{1}+$ $q^{\sigma}(x) \nu^{u}(x), 0 \leq q^{\sigma}(x) \leq q^{u}(x)$.

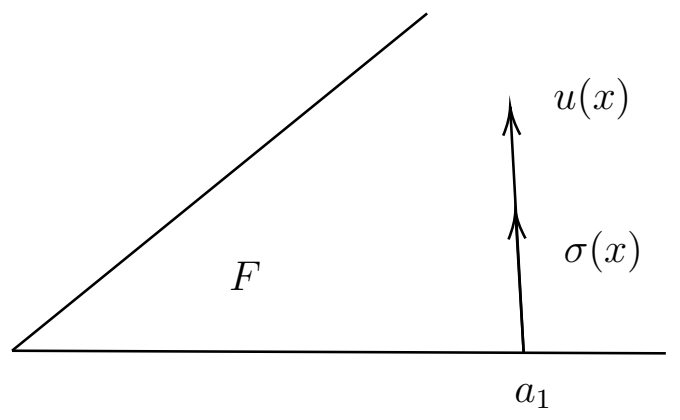

Therefore by the convexity of $F$ the comparison map $\sigma(x)$ is also positive, $\sigma(\bar{F}) \subset \bar{F}$, and it can be extended equivariantly from $F$ to $\mathbb{R}^{n}$ since $B_{R}\left(x_{0}\right) \subset F$ or $B_{R}\left(x_{0}\right) \subset D$ with $x_{0} \in \partial F$, in the boundary of $F$, which consists of reflection planes in $G_{a_{1}}$.

Thus Proposition 1(ii) can be applied for a fixed $q$, with $2 q \leq \rho$, to produce the estimate

$$
B_{C q^{-\alpha}}\left(x_{0}\right) \subset D \Rightarrow u(x) \equiv a_{1} \text { in } B_{\frac{C}{2} q^{-\alpha}}\left(x_{0}\right)
$$


for $C \geq \hat{C}(\alpha, n)$.

By taking a sequence of $C^{\prime} s$ tending to infinity via a covering argument we see that $u(x) \equiv a_{1}$ if $d(x, \partial D) \geq \hat{C}(\alpha, n) q^{-\alpha}$

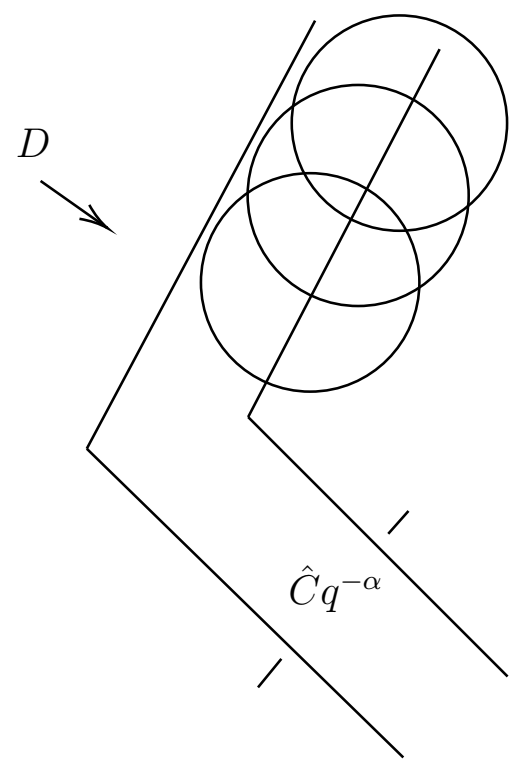

The proof of Theorem 1 is complete.

\subsection{Proof of Proposition 2}

Proof. From the assumption (1.16) and the Basic Estimate we have

$$
\int_{B_{R}\left(x_{0}\right)} \chi_{\left\{u \neq a_{i}\right\}} d x=\int_{B_{R}\left(x_{0}\right)} \chi_{A^{c}}(u) d x \leq C R^{n-1}
$$

But

$$
\int_{B_{R}\left(x_{0}\right)} \chi_{\left\{u \neq a_{i}\right\}} d x=\mathcal{L}^{n}\left(\left\{\left|u-a_{i}\right|>0\right\} \cap B_{R}\left(x_{0}\right)\right)
$$

Hence

$$
\mathcal{L}^{n}\left(\left\{u=a_{i}\right\} \cap B_{R}\left(x_{0}\right)\right) \geq\left|B_{R}\left(x_{0}\right)\right|-c R^{n-1} \geq C R^{n}, R>R_{0} .
$$




\subsection{Proof of Proposition 3}

Proof. Let

$$
0<\theta<d_{0}:=\min \left\{\left|a_{i}-a_{j}\right|: i \neq j, i, j \in\{1, \ldots, N\}\right\}
$$

$\theta$ arbitrary otherwise.

1. We claim that there exist at least two distinct points $a_{i} \neq a_{j}$ in $A$ such that

$$
\mathcal{L}^{n}\left(B_{R}\left(x_{0}\right) \cap\left\{\left|u-a_{k}\right| \leq \theta\right\}\right) \geq C_{k} R^{n} \quad, R \geq R_{0}, k=i, j
$$

$C_{k}=C_{k}(\theta)$.

Proof of the Claim. Since $u$ is a nonconstant minimizer, there is $x_{1}$ such that $u\left(x_{1}\right) \neq a_{1}$

$\Rightarrow \mathcal{L}^{n}\left(B_{\tilde{R}_{0}}\left(x_{1}\right) \cap\left\{\left|u-a_{1}\right|>\lambda\right\}\right) \geq \mu_{0} \quad$ (by continuity, for some $\tilde{R}_{0}, \mu_{0}>0$ and $\lambda>0$ small) and therefore by the Density Estimate (1.24) we have:

$$
\mathcal{L}^{n}\left(B_{R}\left(x_{1}\right) \cap\left\{\left|u-a_{1}\right|>\lambda\right\}\right) \geq c R^{n}, R \geq \tilde{R}_{0} .
$$

Notice that by (3.35), there is $R_{1}\left(x_{0}\right)>0$ such that

$$
\mathcal{L}^{n}\left(B_{R}\left(x_{0}\right) \cap\left\{\left|u-a_{1}\right|>\lambda\right\}\right) \geq c_{1} R^{n}, R \geq R_{1}\left(x_{0}\right) .
$$

Similarly, since $u \neq a_{k}$ there is $x_{k}$ such that $u\left(x_{k}\right) \neq a_{k}$ and we can repeat the arguments above with $x_{k}$ in the place of $x_{1}$ to obtain

$$
\mathcal{L}^{n}\left(B_{R}\left(x_{0}\right) \cap\left\{\left|u-a_{k}\right|>\lambda\right\}\right) \geq c_{k} R^{n}, R \geq R_{k}, k=2, \ldots, N
$$

for some small $\lambda>0$.

By Remark 5.4 in [1], $\forall \lambda_{1}, \ldots, \lambda_{N} \in\left(0, d_{0}\right)$ we have

$$
\mathcal{L}^{n}\left(B_{R}\left(x_{0}\right) \cap\left\{\left|u-a_{k}\right|>\lambda_{k}\right\}\right) \geq c_{k} R^{n}, R \geq R_{0},\left(R_{0}=\max _{k \in\{1, \ldots, N\}} R_{k}\right) .
$$

So, if $\lambda<d_{0}-\theta$ and $\left|u-a_{1}\right| \leq \theta<d_{0} \leq\left|a_{1}-a_{2}\right|$

$$
\Rightarrow\left|u-a_{2}\right| \geq\left|a_{1}-a_{2}\right|-\theta>\lambda>0 \Rightarrow\left\{\left|u-a_{1}\right| \leq \theta\right\} \subset\left\{\left|u-a_{2}\right|>\lambda\right\} .
$$


Thus

$$
\begin{gathered}
A_{2}:=\bigcup_{k=1, k \neq 2}^{N}\left\{\left|u-a_{k}\right| \leq \theta\right\} \subset\left\{\left|u-a_{2}\right|>\lambda\right\} \\
\Rightarrow A_{2} \cup\left[\left\{\left|u-a_{2}\right|>\lambda\right\} \cap A_{2}^{c}\right]=\left\{\left|u-a_{2}\right|>\lambda\right\} \\
\Leftrightarrow A_{2} \cup\left[\left\{\left|u-a_{2}\right|>\lambda\right\} \cap\left(\bigcap_{k=1, k \neq 2}^{N}\left\{\left|u-a_{k}\right|>\theta\right\}\right)\right]=\left\{\left|u-a_{2}\right|>\lambda\right\}
\end{gathered}
$$

and from the Basic Estimate (1.23) and the hypothesis (H1) on $W$ we have

$$
\mathcal{L}^{n}\left(B_{R}\left(x_{0}\right) \cap\left\{\left|u-a_{2}\right|>\lambda\right\} \cap\left(\bigcap_{k=1, k \neq 2}^{N}\left\{\left|u-a_{k}\right|>\theta\right\}\right)\right) \leq \bar{c} R^{n-1}
$$

Hence, by (3.38) and (3.40) it holds

$$
\mathcal{L}^{n}\left(B_{R}\left(x_{0}\right) \cap A_{2}\right) \geq \bar{c}_{2} R^{n} \Leftrightarrow \mathcal{L}^{n}\left(B_{R}\left(x_{0}\right) \cap\left(\bigcup_{k=1, k \neq 2}^{N}\left\{\left|u-a_{k}\right| \leq \theta\right\}\right)\right) \geq \bar{c}_{2} R^{n}
$$

and similarly, if $A_{l}:=\bigcup_{k=1, k \neq l}^{N}\left\{\left|u-a_{k}\right| \leq \theta\right\}, l=1,2, \ldots, N$, we have

$$
\mathcal{L}^{n}\left(B_{R}\left(x_{0}\right) \cap\left(\bigcup_{k \neq l}\left\{\left|u-a_{k}\right| \leq \theta\right\}\right)\right) \geq \bar{c}_{l} R^{n} \quad, R \geq R_{0}
$$

for all $l=1,2, \ldots, N$.

Therefore there exist at least two $i, j \in\{1, \ldots, N\}$ such that

$$
\mathcal{L}^{n}\left(B_{R}\left(x_{0}\right) \cap\left\{\left|u-a_{k}\right| \leq \theta\right\}\right) \geq \bar{c}_{k} R^{n}, R \geq R_{0}, k=i, j,
$$

and the claim is proved.

2. We now proceed to conclude the proof of Proposition 3 ,

Let $\mathcal{A}_{k}^{R}:=\overline{B_{R}\left(x_{0}\right)} \cap\left\{\left|u-a_{k}\right| \leq \theta\right\}, k=i, j$

$$
\begin{gathered}
\int_{\mathcal{A}_{i}^{R}} \chi_{\left\{u \neq a_{i}\right\}}(x) d x=\mathcal{L}^{n}\left(\left\{\left|u-a_{i}\right|>0\right\} \cap \mathcal{A}_{i}^{R}\right) \\
=\mathcal{L}^{n}\left(\bigcap_{k=1}^{N}\left\{\left|u-a_{k}\right|>0\right\} \cap \mathcal{A}_{i}^{R}\right) \quad(\text { by }(\underline{3.39})) \\
\left.=\int_{\mathcal{A}_{i}^{R}} W^{0}(u) d x \leq c R^{n-1} \quad \text { (by the Basic Estimate (1.23) }\right)
\end{gathered}
$$




$$
\begin{gathered}
\mathcal{L}^{n}\left(\left\{u=a_{i}\right\} \cap \mathcal{A}_{i}^{R}\right)=\mathcal{L}^{n}\left(\mathcal{A}_{i}^{R}\right)-\mathcal{L}^{n}\left(\left\{u \neq a_{i}\right\} \cap \mathcal{A}_{i}^{R}\right) \\
\geq c_{i} R^{n}-\mathcal{L}^{n}\left(\left\{u \neq a_{i}\right\} \cap \mathcal{A}_{i}^{R}\right) \quad(\text { by Step 1.) } \\
\left.\geq c_{i} R^{n}-c R^{n-1} \geq C_{i} R^{n}, R \geq R_{0} \quad \text { (by (3.41) }\right)
\end{gathered}
$$

Similarly for $\left\{u=a_{j}\right\}$.

Now, for obtaining (1.20), we utilize the isoperimetric inequality (see for example [22])

$$
\min \left\{\mathcal{L}^{n}\left(\overline{B_{R}\left(x_{0}\right)} \cap E_{i}\right), \mathcal{L}^{n}\left(\overline{B_{R}\left(x_{0}\right)} \backslash E_{i}\right)\right\}^{1-\frac{1}{n}} \leq 2 \hat{c}\left\|\partial E_{i}\right\|\left(B_{R}\left(x_{0}\right)\right)
$$

with $E_{i}=\left\{u(x)=a_{i}\right\} \quad\left(E_{j}=\left\{u(x)=a_{j}\right\}\right)$. Utilizing (1.19), we have

$$
\mathcal{L}^{n}\left(\overline{B_{R}\left(x_{0}\right)} \cap E_{i}\right) \geq c_{i} R^{n}
$$

On the other hand

$$
\overline{B_{R}\left(x_{0}\right)} \backslash E_{i} \supset \overline{B_{R}\left(x_{0}\right)} \cap E_{j}
$$

and once more by (1.19)

$$
\mathcal{L}^{n}\left(\overline{B_{R}\left(x_{0}\right)} \cap E_{j}\right) \geq c_{j} R^{n}
$$

Thus the lower bound (1.20) follows.

The proof of Proposition 3 is complete.

\subsection{Proof of Proposition 4}

Proof. 1. Here we require $N=m+1$ and invoke Lemma 2.9, and thus produce an equivariant, positive minimizer for $\alpha=0$ satisfying the Basic Estimate (3.32). We note that from equivariance and (3.32) it follows that $u \neq$ constant (if $u \equiv$ constant, from equivariance we would have that $u \equiv(0, \ldots, 0)$ which contradicts the Basic Estimate (3.32) since $(0, \ldots, 0) \notin$ $\{W=0\})$. 
2. By Proposition 3 we have that there exist $R_{0}>0$ and at least two distinct $a_{i} \neq a_{j}(i, j \in$ $\{1, \ldots, N+1\})$ such that

$$
\mathcal{L}^{n}\left(B_{R}(0) \cap\left\{u=a_{k}\right\}\right) \geq c_{k} R^{n}, R \geq R_{0}, k=i, j .
$$

We partition $\mathbb{R}^{n}$ in $D^{1}, \ldots, D^{N+1}$ (see (H3)) where in each $D^{i}$ there is a unique global minimum of $W$ (i.e. $a_{i}$, and $D^{1}$ is denoted as $D$ ). Thus $u \neq a_{j}$ in the region $D^{i}(i \neq j)$, so from (3.44) we have

$$
\mathcal{L}^{n}\left(B_{R}(0) \cap\left\{u=a_{i}\right\}\right)=\mathcal{L}^{n}\left(D_{R}^{i} \cap\left\{u=a_{i}\right\}\right) \geq c_{i} R^{n}, R \geq R_{0}, D_{R}^{i}=D^{i} \cap B_{R}(0)
$$

and from the equivariance of $u$ we obtain

$$
\mathcal{L}^{n}\left(D_{R}^{k} \cap\left\{u=a_{k}\right\}\right) \geq c_{k} R^{n}, R \geq R_{0}, k=1, \ldots, N+1 .
$$

3. Finally, from the Basic Estimate (3.32), we have

$$
\mathcal{L}^{n}\left(B_{R}(0) \cap\left(\bigcap_{i=1}^{N+1}\left\{u \neq a_{i}\right\}\right)=\int_{B_{R}(0)} W^{0}(u) d x \leq C R^{n-1}\right.
$$

and therefore

$$
\mathcal{L}^{n}\left(D_{R}^{1} \cap\left\{u \neq a_{1}\right\}\right)=\mathcal{L}^{n}\left(D_{R}^{1} \cap\left(\bigcap_{i=1}^{N+1}\left\{u \neq a_{i}\right\}\right) \leq C R^{n-1} .\right.
$$

The proof of Proposition 4 is complete.

\section{Appendix}

\section{A The Containment}

The following result was established by the first author and P. Smyrnelis in unpublished work [9]. We reproduce it here for the convenience of the reader. For related applications of the method of proof we refer to [38]. 
Proposition 5. (9])

Let $u: \mathbb{R}^{n} \rightarrow \mathbb{R}^{m}$ be a bounded $(|u(x)|<M)$ critical point of the functional

$$
J(u)=\int\left(\frac{1}{2}|\nabla u|^{2}+W(u)\right) d x
$$

in the sense that $\forall \Omega \subset \mathbb{R}^{n}$, open, bounded,

$$
\left.\frac{d}{d \varepsilon}\right|_{\varepsilon=0} J_{\Omega}(u+\varepsilon \phi)=0 \quad, \forall \phi \in C_{0}^{1}(\Omega)
$$

where

$$
W(u)=\left\{\begin{array}{l}
W^{\bar{\alpha}}(u):=\prod_{k=1}^{m+1}\left|u-a_{k}\right|^{\alpha_{k}} \quad, \bar{\alpha}=\left(\alpha_{1}, \ldots, \alpha_{m+1}\right), 0<\alpha_{k} \leq 2 \\
W^{0}(u):=\chi_{\left\{u \in S_{A}\right\}}
\end{array}\right.
$$

and $S_{A}$ defined as the interior of the simplex with vertices $a_{1}, \ldots, a_{m}, a_{m+1}$,

$$
S_{A}:=\left\{\sum_{i=1}^{m+1} \lambda_{i} a_{i} ; \lambda_{i} \in[0,1), \forall i=1, \ldots, m+1, \sum_{i=1}^{m+1} \lambda_{i}=1\right\}
$$

Then

$$
u(x) \in \bar{S}_{A}, x \in \mathbb{R}^{n}
$$

For $\alpha_{k} \in[0,1)$ we require that $u$ in addition is a minimizer in the sense of (1.3), so that (A.5) is available.

Proof. Following an idea from [15] we introduce the set

1. $\alpha_{k} \in(0,1), k=1, . ., m$.

$$
F_{M}:=\left\{u: \mathbb{R}^{n} \rightarrow \mathbb{R}^{m}, u \text { minimizer of } J,|u(x)| \leq M\right\}
$$

By Lemma 2.1 we have the uniform Hölder estimate

$$
|u|_{C^{\beta}\left(\mathbb{R}^{n} ; \mathbb{R}^{m}\right)} \leq C(M) \quad, u \in F_{M}
$$

Let $\Pi$ be the face of the simplex $\bar{S}_{A}$ defined by $a_{2}, \ldots, a_{m+1}$, oppposite to $a_{1}$ and let $e \perp \Pi$. Set

$$
P(u ; x)=\left\langle u(x)-a_{2}, e\right\rangle
$$


where $\langle\cdot, \cdot\rangle$ is the inner product in $\mathbb{R}^{m}$ and the orientation of $e$ is such that $\left\langle a_{2}-a_{1}, e\right\rangle>0$. Set

$$
P_{M}:=\sup \left\{P(u ; x): u(\cdot) \in F_{M}, x \in \mathbb{R}^{n}\right\}
$$

Claim: $P_{M} \leq 0$

Clearly the proposition follows from this claim. We proceed by contradiction. Suppose $P_{M}>0$. Thus there is $\left\{u_{k}\right\} \in F_{M},\left\{x_{k}\right\} \subset \mathbb{R}^{n}$, such that

$$
P_{M}-\frac{1}{k} \leq P\left(u_{k}, x_{k}\right) \leq P_{M}
$$

Set

$$
v_{k}(x):=u_{k}\left(x+x_{k}\right),
$$

and note that $v_{k} \in F_{M}$ and

$$
P_{M}-\frac{1}{k} \leq P\left(v_{k}, 0\right) \leq P_{M}
$$

By (A.5),

$$
\left|v_{k}\right|_{C^{\beta}\left(\mathbb{R}^{n} ; \mathbb{R}^{m}\right)} \leq C(M)
$$

hence by Arzela- Ascoli for a subsequence

$$
v_{k} \stackrel{C^{\beta}}{\rightarrow} v \quad, \text { on compacts }
$$

We have

$$
P(v ; x) \leq P_{M}=P(v ; 0)>0, x \in \mathbb{R}^{n}
$$

By the continuity of $v$ there is $R>0$ such that

$$
\begin{gathered}
\frac{P_{M}}{2} \leq P(v ; x) \leq P_{M}, x \in B(0 ; R) \\
P\left(v_{k} ; x\right)=\left\langle v_{k}(x)-a_{2}, e\right\rangle \geq \frac{P_{M}}{4}, \text { on } B(0 ; R)
\end{gathered}
$$

for $k$ large.

Thus $v_{k}(x)$ uniformly away from $a_{1}, \ldots, a_{m}, a_{m+1}$, we have

$$
\Delta v_{k}-W_{u}\left(v_{k}\right)=0, \text { in } B(0 ; R)
$$


classically, since $W_{u}(u) \in C^{1}$ away from $a_{1}, \ldots, a_{m}, a_{m+1}$ and $x \mapsto W_{u}\left(v_{k}(x)\right)$ Holder by (A.10), thus $u \in C^{2+\beta}(B(0 ; R))$.

We now calculate:

$$
\begin{gathered}
\Delta P=\langle\Delta v, e\rangle=\left\langle W_{u}(u), e\right\rangle \\
\frac{\partial}{\partial v_{j}} W(v)=\frac{\partial}{\partial v_{j}}\left(\prod_{\nu=1}^{m+1}\left|v-a_{\nu}\right|^{\alpha_{\nu}}\right)=\sum_{i=1}^{m+1} \frac{\partial}{\partial v_{j}}\left(\left|v-a_{i}\right|^{\alpha_{i}}\right) \prod_{\nu \neq i}\left|v-a_{\nu}\right|^{\alpha_{\nu}}
\end{gathered}
$$

Notice that

$$
\frac{\partial}{\partial v_{j}}\left(\left|v-a_{i}\right|^{2}\right)^{\frac{\alpha_{i}}{2}}=\alpha_{i}\left|v-a_{i}\right|^{\alpha_{i}-2} \cdot\left(v_{j}-a_{i}^{j}\right)
$$

where $a_{i}=\left(a_{i}^{1}, \ldots, a_{i}^{m}\right)$

Hence

$$
\begin{gathered}
W_{v}(v)=\nabla_{v} W(v)=\sum_{i=1}^{m+1} a_{i}\left(\left|v-a_{i}\right|^{\alpha_{i}-2}\right)\left(v-a_{i}\right) \prod_{\nu \neq i}\left|v-a_{\nu}\right|^{\alpha_{\nu}}= \\
=\alpha_{2}\left|v-a_{2}\right|^{\alpha_{2}-2}\left(v-a_{2}\right) \prod_{\nu \neq 2}\left|v-a_{\nu}\right|^{\alpha_{\nu}}+\sum_{i \neq 2} \alpha_{i}\left|v-a_{i}\right|^{\alpha_{i}-2}\left(v-a_{i}\right) \prod_{\nu \neq i}\left|v-a_{\nu}\right|^{\alpha_{\nu}} .
\end{gathered}
$$

Therefore

$$
\begin{gathered}
\Delta P=\alpha_{2}\left|v-a_{2}\right|^{\alpha_{2}-2} \prod_{\nu \neq 2}\left|v-a_{\nu}\right|^{\alpha_{\nu}}\left\langle v-a_{2}, e\right\rangle \\
+\sum_{i \neq 2} \alpha_{i}\left|v-a_{i}\right|^{\alpha_{i}-2}\left\langle v-a_{i}, e\right\rangle \prod_{\nu \neq i}\left|v-a_{\nu}\right|^{\alpha_{\nu}}
\end{gathered}
$$

Note that by the contradiction hypothesis, $\left\langle v(x)-a_{i}, e\right\rangle>0$ (think of $a_{2}$ as the origin).

Hence $\Delta P>0$ on $B(0 ; R)$ contradicting that $P(v ; x)$ takes its maximum at $x=0$.

2. $\bar{\alpha}=0$

For $W(u)=W^{0}(u):=\chi_{\left\{u \in S_{A}\right\}}$, the proof proceeds similarly. The difference here is that $\Delta P=0$, in $B(0 ; R)$ which also leads to a contradiction by the maximum principle since $P(v ; x)$ takes its maximum at $x=0$.

3. $\alpha_{k} \in[1,2], k=1, \ldots, m$.

In this case we define

$$
F_{M}:=\left\{u: \mathbb{R}^{n} \rightarrow \mathbb{R}^{m}, \Delta u-W_{u}(u)=0,|u(x)| \leq M\right\}
$$


$u$ a weak $W^{1,2}$ solution. By linear elliptic theory we have the estimate (A.5). The rest of the argument is as before.

The proof of the proposition is complete.

\section{B The free boundary}

We follow closely the formal derivation from [1] p.140. We imbed the minimizer in a class of variations, $u(\tau):=u(\cdot, \tau)$, with $u(0)$ corresponding to the minimizer, $u(\tau)=u(0)$ outside a ball $B$ centered at some $x_{0}$ and quite arbitrary otherwise.

Let

$$
U(\tau):=\{|u(\cdot, \tau)-a|>0\}
$$

for

$$
a \in\{W=0\}, u(\tau)=a \text { on } \partial U(\tau)
$$

Set

$$
\lambda(\tau):=\frac{1}{2} \int_{U(\tau)}|\nabla u(\tau)|^{2} d x, \mu(\tau):=\int_{U(\tau)} W(u(\tau)) d x
$$

We denote $V:=\frac{\partial X}{\partial \tau}$ where $X(s, \tau)$ is a parametrisation of $\partial U(\tau), s \in \Omega \subset \mathbb{R}^{n-1}$.

Then we have:

$$
\begin{aligned}
\dot{\lambda}(\tau) & =\int_{U(\tau)} \nabla u(\tau) \nabla u_{\tau}(\tau) d x+\frac{1}{2} \int_{\partial U(\tau)}|\nabla u(\tau)|^{2} V \cdot \nu d S \\
& =\int_{U(\tau)}-\Delta u(\tau) u_{\tau}(\tau) d x+\int_{\partial U(\tau)} \frac{\partial u}{\partial \nu} \cdot u_{\tau} d S+\frac{1}{2} \int_{\partial U(\tau)}|\nabla u(\tau)|^{2} V \cdot \nu d S
\end{aligned}
$$

where $\nu$ is the unit outward normal to $\partial U(\tau)$ (pointing outside $U(\tau)$ ). 
Now from $u(X(s, \tau), \tau)=a$ we obtain:

$$
\begin{aligned}
0 & =\frac{\partial}{\partial \tau}[u(X(s, \tau), \tau)]=\frac{\partial u}{\partial \tau}+\frac{\partial u}{\partial \nu} \frac{\partial X}{\partial \tau} \cdot \nu \\
& =u_{\tau}+\frac{\partial u}{\partial \nu} V \cdot \nu
\end{aligned}
$$

Hence

$$
u_{\tau} \cdot \frac{\partial u}{\partial \nu}=-\left|\frac{\partial u}{\partial \nu}\right|^{2} V \cdot \nu
$$

Then from (B.3) and (B.5) and the equation $\Delta u=W_{u}(u)$ we get:

$$
\dot{\lambda}(0)=\int_{U(0)}-W_{u}(u(0)) u_{\tau}(0) d x-\frac{1}{2} \int_{\partial U(0)}|\nabla u(0)|^{2} V \cdot \nu d S .
$$

On the other hand

$$
\dot{\mu}(\tau)=\int_{\partial U(\tau)} W(u(\tau)) V \cdot \nu d S+\int_{U(\tau)} W_{u}(u(\tau)) u_{\tau}(\tau) d x
$$

Here for $0<\alpha<2$ utilizing that $W(u(0))=0$ on $\partial U(0)$ we get:

$$
\begin{aligned}
0 & =\dot{\mu}(0)+\dot{\lambda}(0) \\
& =-\frac{1}{2} \int_{\partial U(0)}|\nabla u(0)|^{2} V \cdot \nu d S
\end{aligned}
$$

and since $V$ is arbitrary

$$
|\nabla u(0)|=0 \text { on } \partial U(0) \text { for } \alpha \in(0,2) .
$$

(we note that $u \in C^{1, \beta-1}, \beta=\frac{2}{2-\alpha}$ by [8]).

Now, for $\alpha=0$ we have $W(u(0))=1$ on $\partial U(0)$ and

$$
\begin{aligned}
0 & =\dot{\mu}(0)+\dot{\lambda}(0) \\
& =\int_{\partial U(0)} V \cdot \nu d S-\frac{1}{2} \int_{\partial U(0)}|\nabla u(0)|^{2} V \cdot \nu d S
\end{aligned}
$$

hence $\frac{1}{2}\left|\nabla_{+} u(0)\right|^{2}=1$ ( $u$ is only Lipschitz, $\nabla_{+}$is the one-sided gradient $)$. 


\section{References}

[1] Nicholas D. Alikakos, Giorgio Fusco, Panayotis Smyrnelis, Elliptic Systems of Phase Transition Type . Progress in Nonlinear Differential Equations and their Applications, Birkhauser (2018)

[2] Nicholas D. Alikakos, Some basic facts on the system $\Delta u-W_{u}(u)=0$, Proceedings of the American Mathematical Society 139(1), 153-162 (2011)

[3] Nicholas D. Alikakos, A new proof for the existence of an equivariant entire solution connecting the minima of the potential for the system $\Delta u-W_{u}(u)=0$. Commun. Partial Differ. Equ. 37(12), 2093-2115(2012)

[4] Nicholas D. Alikakos, Giorgio Fusco, Density estimates for vector minimizers and application, Discrete Contin. Dynam. Syst. 35(12), 5631-5663 (2015)

[5] Nicholas D. Alikakos, Giorgio Fusco, A maximum principle for systems with variational structure and an application to standing waves, J. Eur. Math. Soc. 17(7), 1547-1567 (2015)

[6] Nicholas D. Alikakos, Giorgio Fusco, Entire solutions to equivariant elliptic systems with variational structure. Arch. Ration. Mech. Anal. 202(2), 567-597(2011)

[7] Nicholas D. Alikakos, Giorgio Fusco, Asymptotic behavior and rigidity results for symmetric solutions of the elliptic system $\Delta u=W_{u}(u)$. Annali della Scuola Normale Superiore di Pisa Nicholas D. Alikakos, Giorgio Fusco, XV(special issue), 809-836(2016)

[8] Nicholas D. Alikakos, Zhiyuan Geng and Arghir Zarnescu, in preparation

[9] Nicholas D. Alikakos and Panayotis Smyrnelis , in preparation

[10] Hans W. Alt, Luis A. Caffarelli and Avner Friedman, Variational problems with two phases and their free boundaries, Trans. Amer. Math. Soc. 282 (1984), 431-461

[11] P.W. Bates, G. Fusco, P. Smyrnelis, Multiphase solutions to the vector Allen-Cahn equation: crystaline and other complex symmetric structures. Arch. Ration. Mech. Anal. 225(2), 685-715(2017)

[12] F. Bethuel, D. Smets, On the motion law of fronts for scalar reaction-diffusion equations with equal depth multiple-well potentials, Chinese Annals of Mathematics, Series B volume 38, p. 83-148(2017)

[13] L. Bronsard, C. Gui, M. Schatzman, A three-layered minimizer in $\mathbb{R}^{2}$ for a variational problem with a symmetric three-well potential, Communications on pure and applied mathematics 49(7), 677-715.

[14] Luis A. Caffarelli, Antonio Cordoba, Uniform convergence of a singular pertubation problem, Commun. Pure Appl. Math. 48, 1-12 (1995) 
[15] Luis A. Caffarelli, Nicola Garofalo, Fausto Segala, A gradient bound for entire solutions of quasi-linear equations and its consequences, Commun. Pure Appl. Math. Vol 47, $1994,1457-1473$

[16] Luis A. Caffarelli, Sandro Salsa, A Geometric Approach to Free Boundary Problems , Graduate Studies in Mathematics Vol 68, 2005; 270 pp;

[17] Luis A. Caffarelli, Henrik Shahgholian, Karen Yeressian, A minimization problem with free boundary related to a cooperative system , Duke Math. J.Volume 167, Number 10 (2018), 1825-1882

[18] Doina Cioranescu and Patrizia Donato, An introduction to homogenization, volume 17 of Oxford Lecture Series in Mathematics and its Applications. The Clarendon Press Oxford University Press, New York 4 (1999): 118.

[19] T. H. Colding and W. P. Minicozzi, A course in minimal surfaces (Vol. 121). American Mathematical Soc. (2011).

[20] M. del Pino, M. Kowalczyk, J. Wei, On De Giorgi's conjecture in dimension $N \geq 9$. Annals of Mathematics, p. 1485-1569, Volume 174 (2011), Issue 3

[21] Serena Dipierro, Alberto Farina, and Enrico Valdinoci, Density Estimates for Degenerate Double-Well Potentials, SIAM J. Math. Anal., 50(6), 6333-6347.

[22] Lawrence C. Evans, Ronald F. Gariephy, Measure theory and fine properties of functions Published April 19, 2015 by Chapman and Hall/CRC

[23] Giorgio Fusco, On some elementary properties of vector minimizers of the Allen-Cahn energy Commun. Pure Appl. Anal. 13(3), 1045-1060 (2014)

[24] Giorgio Fusco , Equivariant entire solutions to the elliptic system $\Delta u-W_{u}(u)=0$ for general G-invariant potentials. Calc. Var. Partial Differ. Equ. 49(3), 963-985 (2014)

[25] Dimitrios Gazoulis, Elliptic Systems with Variational Structure . Can be found online at https://pergamos.lib.uoa.gr/uoa/dl/object/2882475

[26] David Gilbarg, Neil S. Trudinger, Elliptic Partial Differential Equations of Second Order , Springer

[27] C. Gui, M. Schatzman, Symmetric quadruple phase transitions, Indiana Univarsity mathematics journal, 781-836.

[28] Q.Han, F.Lin, Elliptic Partial Differential Equations: Second Edition, American Mathematical Society

[29] D. Mazzoleni, S. Terracini, B. Velichkov, Regularity of the free boundary for the vectorial Bernoulli problem, Anal. PDE 13(3): 741-764 (2020).

[30] L. Modica A Gradient Bound and a Liouville Theorem for Nonlinear Poisson Equations Commun. Pure App. Math. 38(5), 679-684 (1985) 
[31] Di Nezza, Eleonora, Giampiero Palatucci, and Enrico Valdinoci, Hitchhiker's guide to the fractional Sobolev spaces Bulletin des Sciences Mathematiques 136.5 (2012): 521573.

[32] Nguyen, Luc, and Arghir Zarnescu, Refined approximation for minimizers of a Landaude Gennes energy functional Calculus of Variations and Partial Differential Equations 47.1-2 (2013): 383-432.

[33] Arshak Petrosyan, Henrik Shahgholian, Nina Uraltseva, Regularity of Free Boundaries in Obstacle-Type Problems Graduate Studies in Mathematics , Volume 136 (2012)

[34] Pucci, P., Serrin, J.: The strong maximum principle revisited. J. Differential Equations 196, 1-66 (2004)

[35] Pucci, P., Serrin, J.: Dead cores and bursts for quasilinear singular elliptic equations. SIAM J. Math. Anal. 38, No. 1 259-278 (2006)

[36] Pucci, P., Serrin, J.: The maximum principle. Progress in Nonlinear Differential Equations and Their Applications 73, Springer-Birkhäuser (2007)

[37] O. Savin, Regularity of flat level sets in phase transitions. Annals of Mathematics, p. 41-78, Volume 169 (2009)

[38] Panayotis Smyrnelis , Gradient estimates for semilinear elliptic systems and other related results Proceedings of the Royal Society of Edinburgh 145A pp. 1313-1330 (2015)

[39] R.Sperb, Some complementary estimates in the Dead Core problem, Research Report No. 95-05 September 1995

[40] R. Sperb : Some complementary estimates in the dead core problem. Nonlinear Problems in Applied Mathematics. In honor of Ivar Stakgold on his 70th birthday, T. S. Angell, et al. (eds.), Philadelphia, (1996) 217-224.

[41] J. Wei , Geometrization Program of Semilinear Elliptic Equations. AMS/IP Studies in Advanced Mathematics Volume 51, 2012 